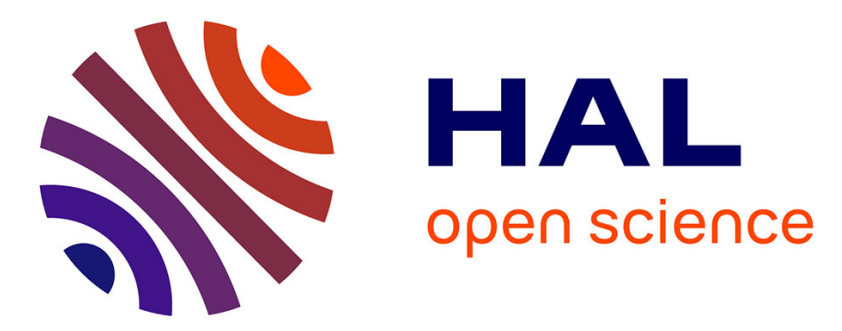

\title{
On the spin-up by a rotating disk in a rotating stratified fluid
}

\author{
F. Y. Moulin, Jan-Bert Flór
}

\section{To cite this version:}

F. Y. Moulin, Jan-Bert Flór. On the spin-up by a rotating disk in a rotating stratified fluid. Journal of Fluid Mechanics, 2004, 516, pp.155-180. 10.1017/S0022112004000655 . hal-00206160

\section{HAL Id: hal-00206160 \\ https://hal.science/hal-00206160}

Submitted on 4 Apr 2020

HAL is a multi-disciplinary open access archive for the deposit and dissemination of scientific research documents, whether they are published or not. The documents may come from teaching and research institutions in France or abroad, or from public or private research centers.
L'archive ouverte pluridisciplinaire HAL, est destinée au dépôt et à la diffusion de documents scientifiques de niveau recherche, publiés ou non, émanant des établissements d'enseignement et de recherche français ou étrangers, des laboratoires publics ou privés. 


\title{
On the spin-up by a rotating disk in a rotating stratified fluid
}

\author{
By F. Y. MOULIN AND J.-B. FLÓR \\ LEGI, BP 53, 38041 Grenoble Cedex 9, France
}

We investigate the response of a rotating stratified fluid to the local spin-up by a small rotating disk of radius $R$, with Rossby number $R o=\omega_{d} / 2 \Omega$ around unity where $\omega_{d}$ is the rotating-disk vorticity and $\Omega$ the background rotation frequency. During an initial stage $\tau_{s u}=O\left(E_{k}{ }^{-1 / 2} N^{-1}\right)$ with Ekman number, $E_{k}=v / \Omega R^{2}(v$ the kinematic viscosity and $N$ the buoyancy frequency), fluid ejected by the Ekman boundary layer mixes with ambient fluid, and forms an intermediate-density intrusion the radial spreading of which is arrested by background rotation. This flow resembles a concentric source-sink configuration with the sink represented by the Ekman layer above the disk and the source by the ejected fluid, which, by conservation of potential vorticity, leads to the formation of a cyclonic vortex embedded in an anti-cyclonic ring. In the next stage, the radial and axial diffusion of momentum dominate the flow evolution, and the flow is characterized by a balance between viscous dissipation of momentum and the amount of momentum applied by the rotating disk. Vorticity diffusion dominates the flow and smooths out the flow history when $E_{k}^{-1 / 2}(f / N)<3$, whereas the initial stage can be recognized as a separate flow stage when $E_{k}^{-1 / 2}(f / N)>3$. The stability of the density front is discussed.

\section{Introduction}

The spin-up of rotating stratified fluids is relevant to the dynamics of many geophysical flows. Some examples are surface-wind-driven flows, currents in coastal regions and spin-up in closed basins and geophysical vortical flows. This paper is most relevant to the latter, the spin-up processes that play a role in the dynamics of ocean and atmospheric vortices; for example, meddies (McWilliams 1985), arctic eddies (Manley \& Hunkins 1985), megaplume hydrothermal emissions (Baker 1995) and in the atmosphere the polar vortex. In this context we investigate the response of a rotating stratified fluid to the local spin-up by a small rotating disk. The spin-up of stratified fluids has been considered for cylindrical containers, rectangular and conical containers, and for horizontally infinite rotating disks (for a review see Duck \& Foster 2001), but the local spin-up, such as generated by a small disk, has not previously been explored in detail before.

The closest experimental studies to the present investigation are those of Baker (1971) and Calman (1977) who employed a small rotating disk at mid-depth in a rotating stratified fluid to investigate McIntyre's (1970) double-diffusive instability. For Prandtl number $P r=v / \kappa$ larger than 1 (with $v$ kinematic viscosity and $\kappa$ salt diffusivity), momentum diffuses faster than density leading to a convective instability for Richardson numbers, $R i=N^{2} /(\partial u / \partial z)^{2}<\operatorname{Pr} / 4$, with $N$ the buoyancy frequency, $\partial u / \partial z$ the vertical shear and $\operatorname{Pr} / 4=175$ for a saltwater solution. These studies do not 
consider the spin-up of the flow near the disk, or the ejection of fluid and formation of a front at the disk edge.

Based on thermal wind balance and hydrostatic balance, the vortex that will eventually result from the spin-up process above the disk will take a lenticular shape of aspect ratio $H / R=a f / N$, with $H$ the half-height and $R$ the radius of the vortex and $a$ a constant that depends on the Rossby number, $R o=\omega_{d} / f$, where $\omega_{d}$ the disk vorticity and $f$ the Coriolis parameter (see (4.6) in $\S 4.1$ ). In the limit of no stratification, Ekman pumping above and below the disk stretches and squeezes the fluid over the entire depth leading to a Taylor column. Hide \& Titman (1967) explored this flow, which is controlled by the Ekman number and the disk's relative rotation speed, to investigate the detachment of vertical shear layers and the development of non axisymmetric modes. In the limit of no background rotation, the ejected mixed fluid expands unbounded to the outer boundary, setting up a meridional circulation over the horizontal width of the tank. Davies et al. (1995) used a disk of the tank size that rotated at the bottom of a stratified fluid to investigate the influence of the shear stress and the relative importance of secondary circulation and mixing (see also Spence, Foster \& Davies 1992). They also explored the mixing by a small rotating disk. In the presence of background rotation, the fluid ejected by the Ekman boundary is limited, and in geostrophic flows it expands till it reaches the deformation radius where the front speed equals the velocity induced by the Coriolis force.

When fluid is spin-up by a small rotating disk, the Ekman layer acts as a suction pump, with the disk surface the sink, and the edge the source. Most likely because of the nonlinear dynamics of the flow induced by this ejected fluid at the disk edge, there are no analytical treatments of this particular flow. Kloosterziel (2000) analyses inertia-gravity waves and geostrophic vortices generated by suction or pumping forcing at an upper rigid surface; the same forcing as one would achieve by means of a rotating disk and the consequent Ekman pumping or suction. Though that study focuses mainly on the radiation of waves, it contains interesting aspects that are relevant to the present study: Kloosterziel (2000) shows that in addition to waves, a vortex flow is eventually generated by this forcing, with an aspect ratio proportional to $f / N$. However, his approach is constrained to axisymmetric inviscid flows, and the further evolution driven by viscous diffusion of momentum is not considered. This latter aspect of the flow evolution was considered analytically by Flierl $\&$ Mied (1985) who studied the related problem of the spin-down of a warm core ring in a continuously stratified fluid with viscous friction at the surface, including vertical and horizontal diffusion of momentum and buoyancy. Based on their choice of horizontal and vertical mixing parameters, they find that horizontal diffusion dominates the spin-down while Ekman down-welling acts only in the layer near the surface; vertical diffusion is found ineffective in driving azimuthal circulation. They conclude that the spin-down is a consequence of horizontal diffusion and the resulting meridional circulation. In contrast with their study, vertical and horizontal parameters for the momentum diffusion are equal in the present work, and the diffusive evolution is also driven by the rotating disk which provides momentum to the flow. The meridional circulation associated with this diffusive evolution may be quantitatively different.

We show that the initial stage of the spin-up is similar to a source-sink configuration driven by the circulation through the Ekman layer at the disk surface. This flow is well-predicted by a model based on conservation of potential vorticity. The next stage is characterized by a diffusive evolution that gradually smooths out the initial vorticity distribution generated by stretching. Since the meridional circulation induced by diffusive processes generally slows down the spreading of momentum, the diffusive evolution of this stage is overestimated by a pure diffusion model. 


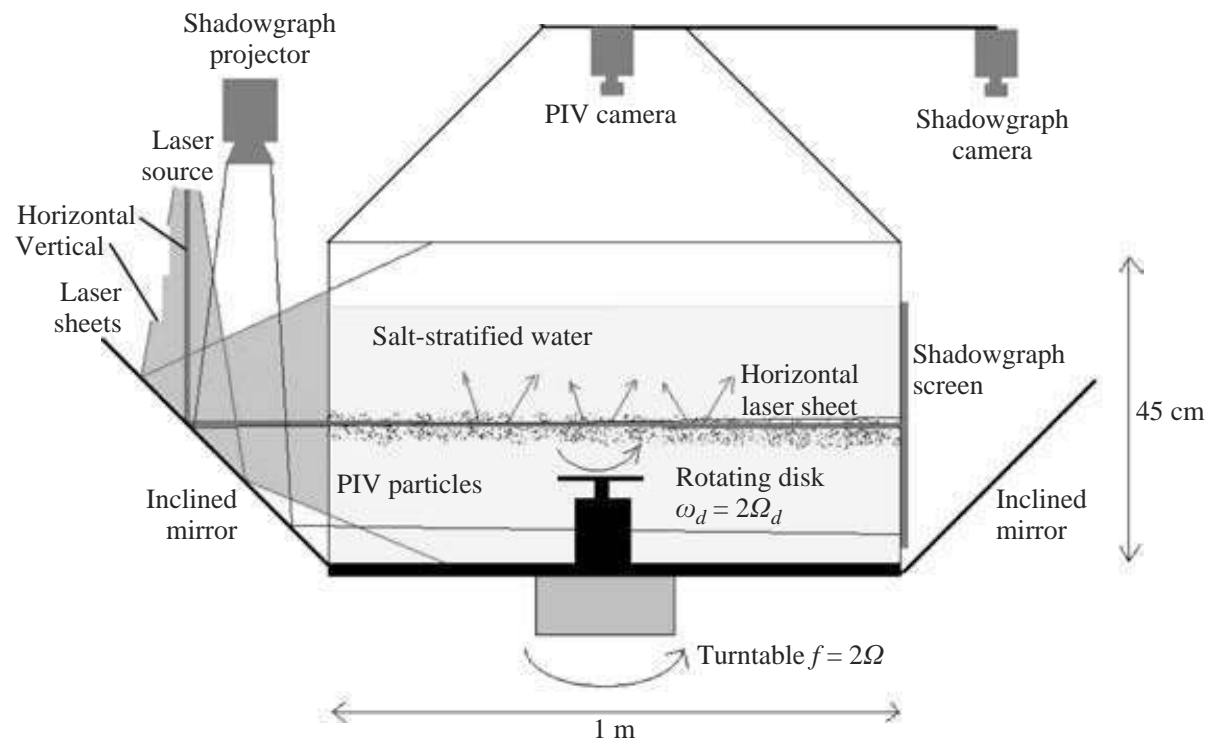

FIGURE 1. Sketch of the experimental set-up.

In $\S 2$, we describe experimental methods, followed by the experimental observations of the different stages in $\S 3$. In $\S 4$, we present the models of the initial stage of the spinup and the subsequent diffusive evolution, followed by a discussion of the modelling of instabilities observed in the experiments. Comparisons of the predictions of these models with the experimental results are presented in $\S 5$. Conclusions are drawn in $\S 6$.

\section{Experimental methods}

Experiments were carried out in a $1 \mathrm{~m}$ square tank of $45 \mathrm{~cm}$ depth, mounted on a rotating table. A side view of the experimental set-up is sketched in figure 1. The tank was filled with a linearly salt-stratified fluid rotating with a uniform angular velocity $\Omega$ about its vertical axis. The Coriolis parameter $f=2 \Omega$ varied between $0.13 \mathrm{rad} \mathrm{s}^{-1}$ and $0.7 \mathrm{rad} \mathrm{s}^{-1}$, and the buoyancy frequency $N$ of the salt solution, defined as $N^{2}=$ $-\left(g / \rho_{o}\right)(\partial \rho / \partial z)$ (with $g$ the gravitational acceleration, $\rho$ and $\rho_{o}$ the density variation and mean density respectively), was taken between $1.2 \mathrm{~s}^{-1}$ and $1.6 \mathrm{~s}^{-1}$. Experimental values of $N$ were obtained from samples taken at different depths, the density of which was measured with a refractometer, or by recording vertical density profiles with a conductivity probe. The fluid was spun-up by a horizontal disk of radius $R(R=6 \mathrm{~cm}$ or $R=12 \mathrm{~cm}$ and thickness $3 \mathrm{~mm}$ ) placed at mid-height near the rotation axis of the turntable and mounted on the axis of a motor embedded in a waterproof plastic cylinder. The distance from the tank base to the lower surface of the disk was $20 \mathrm{~cm}$. The disk rotated about a vertical axis in the cyclonic direction with a vertical vorticity $\omega_{d}$ ranging from $0.13 \mathrm{rad} \mathrm{s}^{-1}$ to $0.6 \mathrm{rad} \mathrm{s}^{-1}$. The value of the Rossby number $R o=\omega_{d} / f$ was varied between 0.3 and 3.3 whereas the disk Reynolds number, $R e=\omega_{d} R^{2} / \nu$, was between 320 and 3700 .

To measure the horizontal velocity field in horizontal layers above the disk, the motion of tracer particles of diameter $20 \mu \mathrm{m}$, seeded in the flow and illuminated from the side by a horizontal laser sheet, was recorded with a B\&W CCD camera from the top. This camera was fixed to the rotating table and the images were written to the hard disk of a PC mounted on the rotating table. The horizontal velocity fields were 


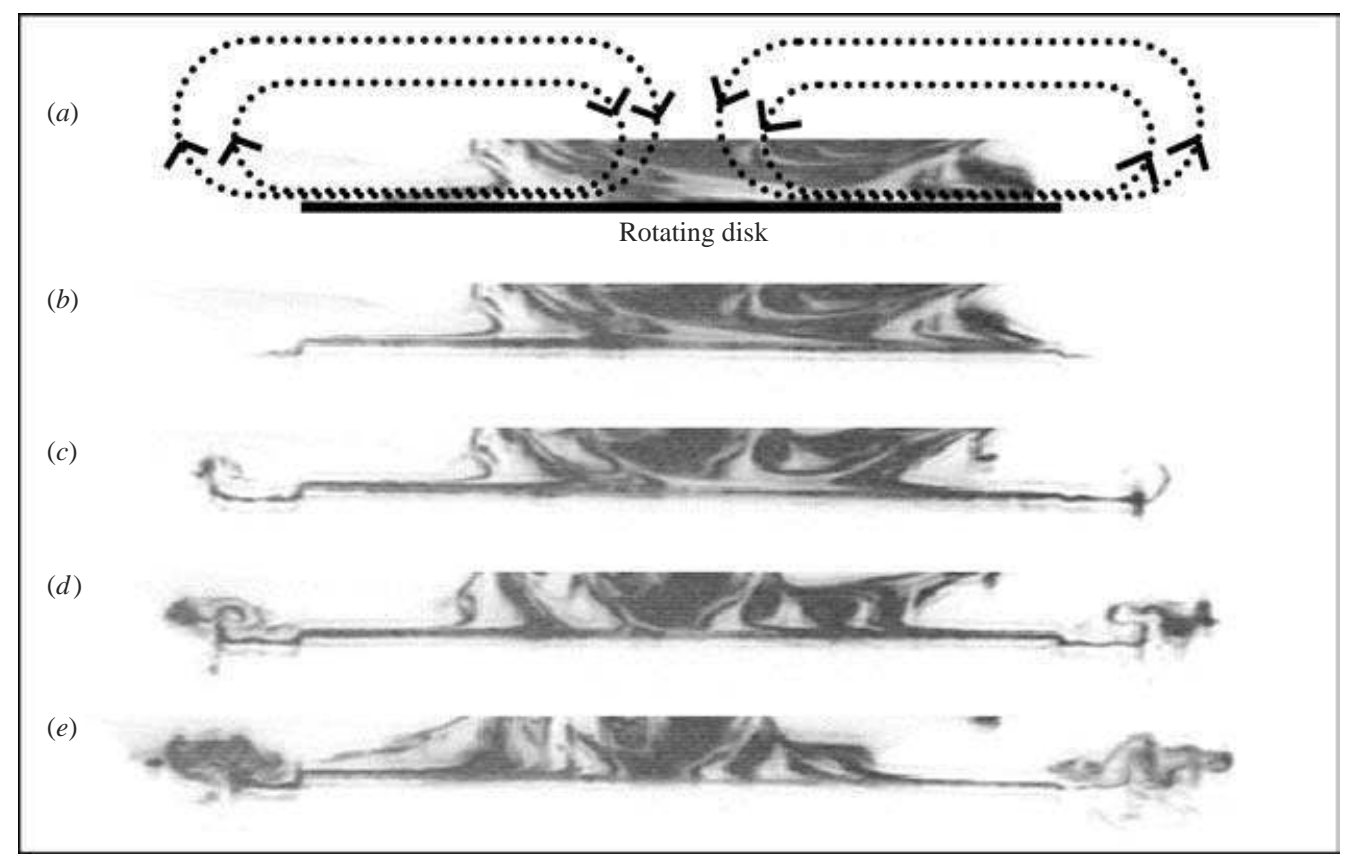

FIGURE 2. Side-view visualization of the flow near the disk after the onset of the disk rotation at $t=0$, showing the Ekman transport and the formation of the density front. Fluoroscein dye was introduced above the rotating disk before $t=0$, and is illuminated by a vertical laser sheet across the disk centre. The experimental parameters are: $f=0.5 \mathrm{~s}^{-1}, N=1.5 \mathrm{~s}^{-1}, \omega_{d}=1.0 \mathrm{~s}^{-1}$, $R=12 \mathrm{~cm}$, and $(a) t=2.4 \mathrm{~s},(b) 7.2 \mathrm{~s},(c) 12 \mathrm{~s},(d) 17 \mathrm{~s}$ and $(e) 22 \mathrm{~s}$ after $t=0$. A sketch of the meridional circulation induced by the transport through the Ekman boundary layer is added to $(a)$.

then calculated by means of particle image velocimetry (PIV) techniques developed by Fincham \& Spedding (1997) and Fincham \& Delerce (2000). In addition, the flow near the disk was visualized with fluorescein dye, released in the neighbourhood of the disk, or with thin white powder produced by the precipitation method of Honji, Taneda \& Tatsudo (1980). The latter method is based on the electrolytic generation of tiny particles by a solder wire used as an electrode, here attached to the edge of the disk. To visualize the vertical motions and the meridional circulation, the fluorescein dye or the solder particles were illuminated by a vertical laser sheet. The dye advection and the instabilities were visualized using a horizontal laser sheet.

Density fluctuations were detected by the shadowgraph method or by the vertical displacements of horizontal layers of fluorescein dye, illuminated with a vertical laser sheet. These horizontal layers were produced before the experiment began by a grid of horizontal dyed wires, using the method employed by Hopfinger et al. (1991) and Flór, Ungarish \& Bush (2002). An example of the final state of the spin-up flow is shown in figure $5(a)$.

\section{Observations}

\subsection{Initial spin-up}

After the onset of the disk rotation, fluid in a thin boundary layer is brought into rotation and ejected centrifugally outwards, driving a radial and vertical circulation (see figure 2). The outward transport near the disk surface is opposed by the vertical 
(a)

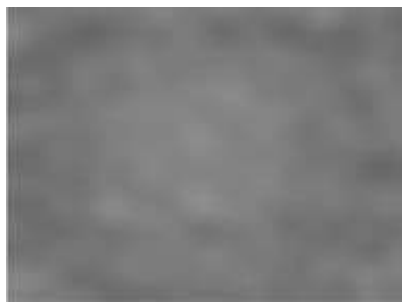

(d)
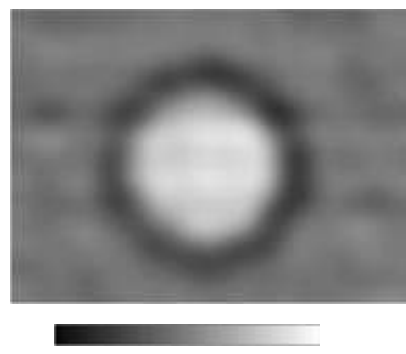

$-0.1 \mathrm{~s}^{-1}$ (b)

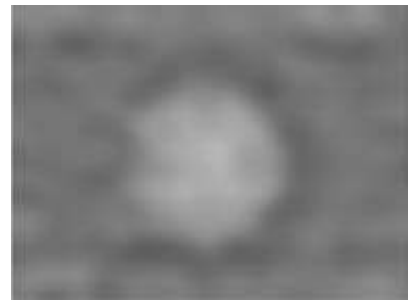

(e)

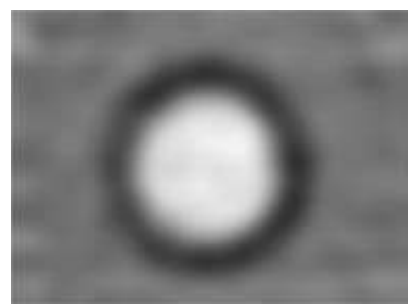

(c)

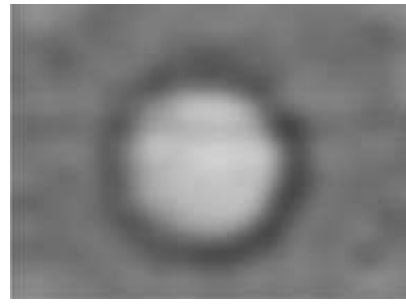

$(f)$

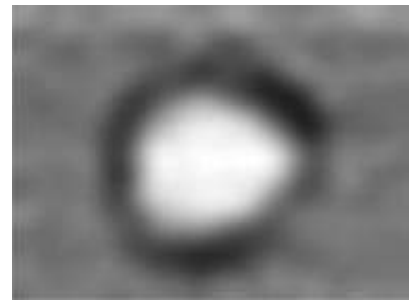

FIGURE 3. Time sequence of the vertical vorticity field from PIV measurements in an horizontal plane $2 \mathrm{~cm}$ above the disk showing the generation of cyclonic vorticity by stretching of background vorticity and the formation of an anticyclonic ring above the growing density front for $\Delta t=50.0$. Experimental parameters are: $f=0.26 \mathrm{~s}^{-1}, N=1.4 \mathrm{~s}^{-1}, \omega_{d}=0.24 \mathrm{~s}^{-1}$, $R=12 \mathrm{~cm}$, and $(a) t=0,(b) 50,(c) 100,(d) 150,(e) 200$ and $(f) 250 \mathrm{~s}$.

pumping of the fluid above the disk, whereas the fluid ejected at the edge of the rotating disk generates a circular density front which spreads out vertically and radially. In a vertical plane, streamlines of this meridional circulation then form closed loops with a downward direction in the region above the rotating disk and an upward direction in the region where the density front is generated (see figure $2 a$ ).

The boundary layer is formed after a few disk rotations and is of thickness a few milimetres, in agreement with previous analytical and numerical studies (see e.g. Greenspan 1968) which have shown that, even for values of the Rossby number close to unity, the thickness of such boundary layers is the same as that of Ekman layers based on the geostrophic approximation, and given by $\sqrt{2 v / f}$. The density front forms at the edge of the disk and is generated by mixing of the ejected fluid with ambient fluid at rest. It was observed from the motion of solder particles introduced at the edge of the disk that the fluid at the front acquired anticyclonic azimuthal velocity whereas the fluid close to the disk was transported by the Ekman layer and acquired cyclonic azimuthal velocity. Therefore, the nose of the density front is moving in the anticyclonic direction, with azimuthal velocity growing and becoming cyclonic when approaching the edge of the disk.

In the region above the disk, the Ekman suction stretches the background vorticity, thus generating cyclonic vorticity. Figure 3 shows a time sequence of the vertical vorticity field from measurements in a horizontal plane above the rotating disk. An anticyclonic ring is also generated in the region above the density front, associated with the compression of the background vorticity, thus generating anticyclonic vorticity.

Away from the boundary layer and the density front, this initial flow is close to that induced by a time-dependent source-sink configuration like the one studied by 


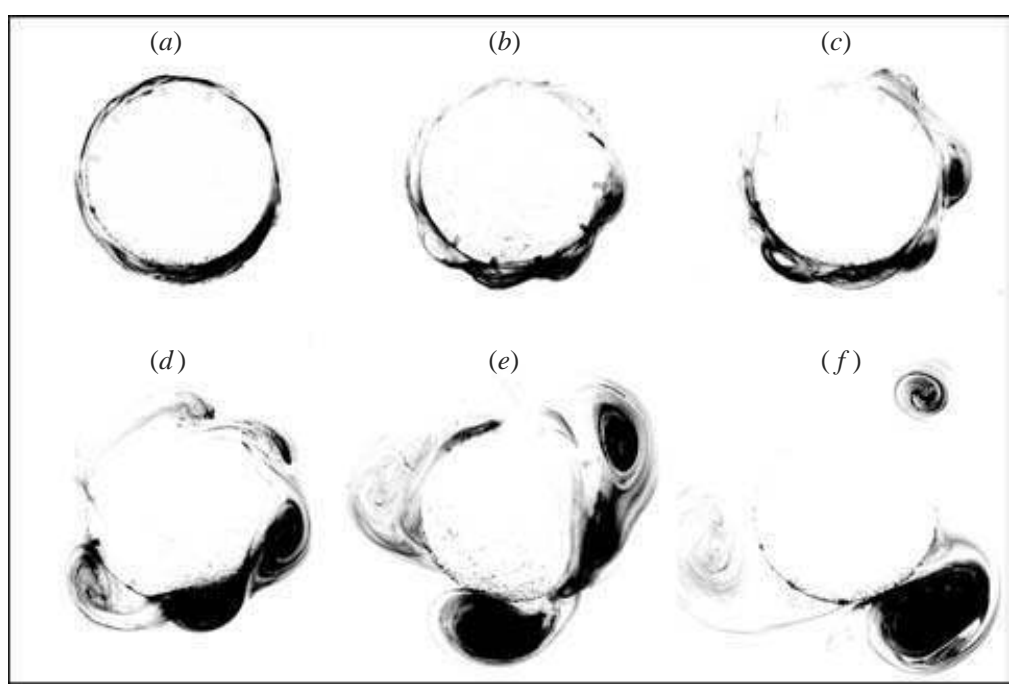

FIGURE 4. Top view visualization of the baroclinically unstable density front. The flow is visualized by solder particles, generated at the rim of the disk before the onset of the disk rotation, and illuminated by a horizontal laser sheet located just above the disk for $(a) t=28 \mathrm{~s}$, (b) $47 \mathrm{~s},(c) 66 \mathrm{~s},(d) 112 \mathrm{~s},(e) 164 \mathrm{~s}$ and $(f) 260 \mathrm{~s}$. The experimental parameters are: $f=0.6 \mathrm{~s}^{-1}$, $N=1.5 \mathrm{~s}^{-1}, \omega_{d}=0.3 \mathrm{~s}^{-1}, R=12 \mathrm{~cm}$.

Kloosterziel (2000) in the geostrophic limit: the sink is associated with the Ekman suction; the source corresponds to the generation of the density front and its spreading. As the vorticity of fluid near the disk surface increases, the suction speed driven by the Ekman boundary layer decreases proportionally. Therefore, the growth rate of the vortex generated by the Ekman circulation decreases with time.

\subsection{Non-axisymmetric and long-time evolution}

Generally, the density front generated by the ejected spun-up fluid is found to be unstable and wave-like irregularities appear in a manner similar to that shown by Griffith \& Linden (1981) for an annular source flow in a homogenous fluid, or Bush \& Woods (1999) for a line plume in a stratified rotating fluid. As the instability develops (see figure 4), the anticyclonic ring breaks up into satellite vortices which merge and eventually evolve into a pattern with usually two or three anticyclonic satellites. At this stage, the cyclonic core is torn apart by the strain generated by the anticyclonic vortices, which themselves diffuse laterally. Depending on the strengths of the satellites relative to the vortex core and their diffusive spreading, the vortex breaks up into two dipoles or gradually returns to an axisymmetric state. In the former case, the dipoles propagate away from the disk and the same spin-up sequence repeats, perturbed by the flow of the surrounding dipoles. In the latter case, vertical and azimuthal diffusion of vorticity smooths azimuthal variations, leading to an almost Gaussian vorticity profile at large distances from the disk.

\section{Modelling}

Following the observations described in the previous section, the flow around the rotating disk can be divided into three regions, as illustrated in figure 5(b). Region I corresponds to the Ekman layer of thickness $\delta=\sqrt{2 v / f}$ where the fluid is transported 
(a)

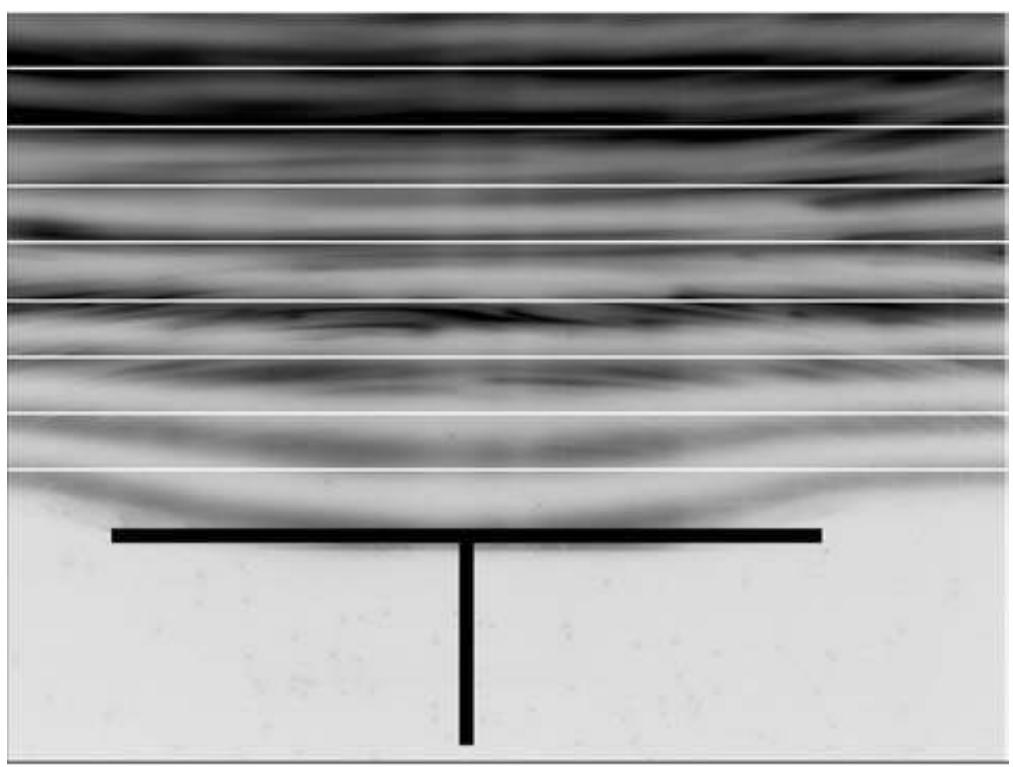

(b)

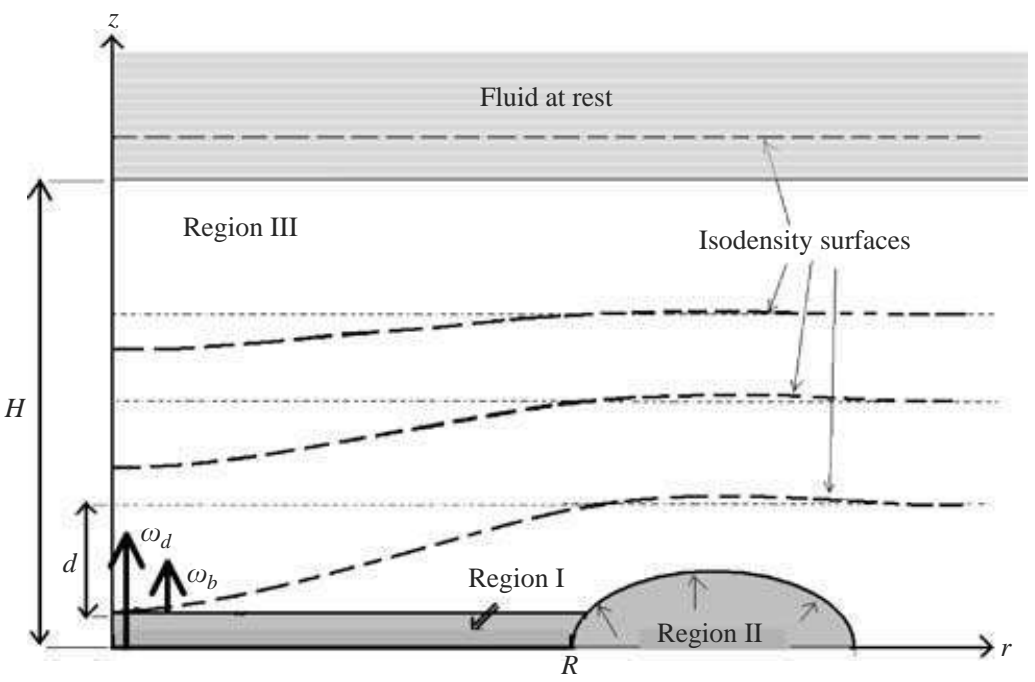

FIGURE 5. (a) Side-view dye visualization of the spin-up flow in its final state, illustrating the deviation of the density field. The black streaks represent the fluoresceine dye planes illuminated by a vertical laser plane at $t=48 \mathrm{~min}$, whereas the white lines represent their initial position at $t=0$ (colours in the image are inverted). (b) Schematic of the flow for the stretching model, with region I the boundary layer, region II the density front generated by ejected fluid of region I, and region III, where fluid is spin-up by stretching and compression of background vorticity, extending to a limited height $H$ because of the stratification. $\omega_{b}$ and $\omega_{d}$ are the vertical vorticity at the base of region I and of the rotating disk, respectively.

radially outwards. Region II corresponds to the density front generated by the fluid ejected at the edge of the disk. From now, we will focus on the flow in region III, away from the boundary layer and the density front, and limited in the vertical direction. 
The vertical extent of region III, $H$, depends on the process driving the flow evolution. Above region III, the fluid is at rest.

The observations showed that the transport of fluid along the Ekman boundary layer leads to a descent of isodensity surfaces above the disk and generates the density front. Therefore, the flow in region III can be interpreted as that arising from a sink located on the disk surface and a source located at the edge of the disk. The strength of this source-sink configuration is given by the Ekman suction velocity at the top of the boundary layer, and is thus proportional to the difference in rotation between the base of the flow in region III and the rotating disk. In a first approximation, the viscous effects in region III can be neglected and a relationship between the displacement of the isodensity surfaces and the vorticity generation can be established using conservation of absolute potential vorticity. This approach will be presented in detail in the next subsection as the stretching model and will provide a typical timescale $\tau_{s u}$ for the local spin-up by vortex stretching as well as a typical vertical lengthscale $H$ for the vortex eventually generated.

During the entire evolution, the flow in region III exhibits vertical and horizontal shear, giving rise to diffusive transport of momentum. This transport is described by a separate model, the diffusive model, based on the assumption that the flow evolution in region III is fully driven by momentum diffusion from the rotating disk, thus neglecting the generation of vorticity by stretching of the background vorticity.

Both processes, stretching of background vorticity and diffusion, contribute to the generation of momentum in region III. During an initial flow stage, Ekman suction results in vortex stretching on a vertical scale $H$ and a timescale $\tau_{s u}$. In a subsequent stage, the flow is dominated by the radial and vertical diffusion of momentum with a diffusive timescale $\tau_{\text {diff. }}$. When $\tau_{\text {su }}<\tau_{\text {diff, }}$, the separation between these two successive stages is easily observed in the experimental measurements. For $\tau_{d i f f}<\tau_{s u}$, this separation becomes meaningless and the spin-up of fluid in region III is driven by diffusive processes. For the two cases, on timescales $t \gg \tau_{\text {diff }}$, momentum diffusion will govern the flow evolution and smooth out any initial vorticity distribution generated by vortex stretching. The stretching model (in $\S 4.1$ ) and diffusive model (in $\S 4.2$ ) proposed below will allow us to determine the relative contribution of the two processes, stretching and diffusion, during the flow evolution.

Of course, the symmetry-breaking instability of the density front (represented by region III) cannot be described in the framework of the axisymmetric stretching and diffusive models. These models are also unable to reproduce the formation, for very large times $\left(t \gg \tau_{d i f f}, \tau_{s u}\right)$, of layers in shadowgraph images of region II. These two instabilities require separate modelling, which will be presented in the last subsection (in $\S 4.3$ ).

\subsection{Stretching model}

We consider an inviscid evolution from rest for the flow in region III, the dynamics being driven by the Ekman transport in region I. In accordance with experimental observations showing a slow Ekman suction, we will neglect the time-dependent terms in the momentum equation. Then, the flow in region III can be fully described by an equation expressing the balance among the strengths of the centrifugal force, the Coriolis force and the buoyancy force, and an equation expressing the conservation of the potential vorticity from rest. To solve these two equations, boundary conditions at the bottom of region III have to be specified.

The only known boundary condition at the bottom of region III is a dynamical one, expressed as the continuity of the vertical velocity between the top of the Ekman 
boundary layer and the bottom of region III. Knowing a stationary flow solution in region III, it is straightforward to calculate the vertical flux of fluid through the bottom boundary. Therefore a dynamical coupling can be established between a family of stationary flow solutions in region III and the Ekman layer dynamics in order to describe the flow evolution.

In this subsection, we will first solve the set of equations governing the flow in region III for some specific boundary conditions. We will then deduce the flux of fluid through the bottom boundary for this family of stationary solutions. Finally, we will deduce the flux evolution with time from the Ekman vertical velocity and use this result to obtain the time dependence of the flow in region III as well as a typical spin-up timescale $\tau_{\text {su }}$.

\subsubsection{Stationary flow solutions}

In order to describe the spin-up process step by step, we now consider stationary flow solutions for the fluid in region III. Let $\omega_{b}$ define the vertical vorticity at the base of the flow and $\omega_{d}$ the vertical vorticity scale on the disk surface, as above. We consider a fluid of density $\rho$ satisfying the Boussinesq condition, in a cylindrical coordinate system $(r, \theta, z)$, the origin of which is located at the centre of the rotating disk. The difference between the density $\rho$ and the basic state is represented by a linear density profile $\bar{\rho}(z)$ and a deviation from this profile $\tilde{\rho}$, so that

$$
\rho(r, z)=\rho_{o}+\bar{\rho}(z)+\tilde{\rho}(r, z) .
$$

The velocity field in the final flow of region III is uniquely prescribed by its azimuthal component $v$. The conservation of potential vorticity (Ertel's theorem) between the initial state (fluid at rest) and the present stationary state is written

$$
\nabla \rho \cdot \omega_{a}=\frac{\partial \bar{\rho}}{\partial z} f
$$

where $\omega_{a}=\omega_{z}+f \boldsymbol{e}_{z}$ is the absolute vorticity and $\omega_{z}=(\partial v / \partial r+v / r) \boldsymbol{e}_{z}$ the relative vertical vorticity, and becomes

$$
\left(\frac{\partial \bar{\rho}}{\partial z}+\frac{\partial \tilde{\rho}}{\partial z}\right)\left(f+\frac{\partial v}{\partial r}+\frac{v}{r}\right)+\left(\frac{\partial \tilde{\rho}}{\partial r}\right)\left(-\frac{\partial v}{\partial z}\right)=-\frac{\rho_{o}}{g} N^{2} f .
$$

Denoting as $\omega_{b}$ the typical value of the vertical vorticity at the bottom of region III and scaling the equation above with $(r, z) \rightarrow(R r,(f R / N) z)$ and $(v, \tilde{\rho}) \rightarrow$ $\left(\left(\omega_{b} R / 2\right) v,\left(\rho_{o} / g\right) N \omega_{b} R \tilde{\rho}\right)$, yields

$$
\left(1-R o_{b} \frac{\partial \tilde{\rho}}{\partial z}\right)\left(1+\frac{R o_{b}}{2} \frac{1}{r} \frac{\partial(r v)}{\partial r}\right)+\frac{R o_{b}^{2}}{2}\left(\frac{\partial v}{\partial z} \frac{\partial \tilde{\rho}}{\partial r}\right)=1
$$

with $R o_{b}$ the Rossby number, defined as $R o_{b}=\omega_{b} / f$, so that the thermal wind balance can be represented by

$$
\frac{1}{2}\left(1+R o_{b} \frac{v}{r}\right) \frac{\partial v}{\partial z}=-\frac{\partial \tilde{\rho}}{\partial r} .
$$

Integration of (4.2) along the radial coordinate, using the fact that $\tilde{\rho}$ vanishes at infinite radius, yields

$$
\tilde{\rho}(r, z)=\frac{1}{2} \int_{r}^{\infty} \frac{\partial v}{\partial z}\left(1+R o_{b} \frac{v}{u}\right) \mathrm{d} u
$$


Using (4.2) to eliminate the radial derivative of the density perturbation in (4.1), we find an equation for the vertical derivative of the density perturbation:

$$
\left(1-R o_{b} \frac{\partial \tilde{\rho}}{\partial z}\right)=\frac{1+\frac{R o_{b}^{2}}{4}\left(1+R o_{b} \frac{v}{r}\right)\left(\frac{\partial v}{\partial z}\right)^{2}}{1+\frac{R o_{b}}{2} \frac{1}{r} \frac{\partial(r v)}{\partial r}} .
$$

Differentiation of (4.3) along the $r$-coordinate and (4.2) along the $z$-coordinate yields a set of two equations with identical density perturbation terms $\partial^{2} \tilde{\rho} / \partial r \partial z$. After some manipulations, including multiplication by $\left(1+R o_{b} / 2(v / r+\partial v / \partial r)\right)^{2}$ and division by $R o_{b} / 2$, this leads to the nonlinear differential equation for the azimuthal velocity

$$
\begin{aligned}
& \left(\frac{R o_{b}}{r}\left(\frac{\partial v}{\partial z}\right)^{2}+\left(1+R o_{b} \frac{v}{r}\right) \frac{\partial^{2} v}{\partial z^{2}}\right)\left(1+\frac{R o_{b}}{2}\left(\frac{v}{r}+\frac{\partial v}{\partial r}\right)\right)^{2}-\frac{R o_{b}}{2} \\
& \quad \times\left[2\left(1+R o_{b} \frac{v}{r}\right) \frac{\partial v}{\partial z} \frac{\partial^{2} v}{\partial r \partial z}+R o_{b}\left(-\frac{v}{r^{2}}+\frac{1}{r} \frac{\partial v}{\partial r}\right)\left(\frac{\partial v}{\partial z}\right)^{2}\right]\left(1+\frac{R o_{b}}{2}\left(\frac{v}{r}+\frac{\partial v}{\partial r}\right)\right) \\
& \quad+\left(1+\frac{R o_{b}^{2}}{4}\left(1+R o_{b} \frac{v}{r}\right)\left(\frac{\partial v}{\partial z}\right)^{2}\right)\left(-\frac{v}{r^{2}}+\frac{1}{r} \frac{\partial v}{\partial r}+\frac{\partial^{2} v}{\partial r^{2}}\right)=0 .
\end{aligned}
$$

To solve this equation, we have to choose the boundary conditions for $r<1$ as well as for $r>1$. For $r<1$, the vorticity on the disk surface at the end of the spin-up process, $\omega_{b}$, must be constant and equal to the disk vorticity $\omega_{d}$. For $r>1$ the boundary condition is more difficult to define: the vertical vorticity is negative but neither the radial extent of this anticyclonic ring nor its vorticity profile are known, since they depend on the spreading of the density front ejected at the edge of the disk. To investigate the influence of the radial extent of the anticyclonic ring on the flow structure, we considered an isolated vortex with a cyclonic core, embedded in an anticyclonic ring, both with a constant vorticity. We solved equation (4.4) for three different ring sizes, $L=0.5, L=1.0$ and $L=1.5$, and found its influence to be weak (see the Appendix for further discussion on the effect of $L$ and on the numerical method used in solving (4.4)). For simplicity, we present the numerical results only for $L=1.0$.

In the geostrophic limit of $R o_{b}=0,(4.4)$ is linear, and becomes

$$
\frac{\partial^{2} v}{\partial z^{2}}-\frac{v}{r^{2}}+\frac{1}{r} \frac{\partial v}{\partial r}+\frac{\partial^{2} v}{\partial r^{2}}=0
$$

It can be solved by a numerical relaxation method or by Hankel transform of the boundary conditions (see e.g. Clercx et al. 2001). Complete agreement was found for the solutions obtained by the two methods, and the vertical extent of the vortex flow solution was in good agreement with the results of Kloosterziel (2000), who studied the generation of waves and vortices by a similar source-sink configuration at an upper rigid surface of a rotating stratified fluid. Further comparison between our flow solutions and his analytical solutions could not be made since the boundary conditions are different.

For the ageostrophic balanced states, associated with finite values of $R o_{b}$, the vertical extent of the vortex increases with $R o_{b}$ (see figure 6). The nonlinear term $R o_{b}(v / r)(\partial v / \partial z)$ associated with the centrifugal force in the thermal wind equation (4.2) amplifies the impact of the velocity shear on the density field. Since the 

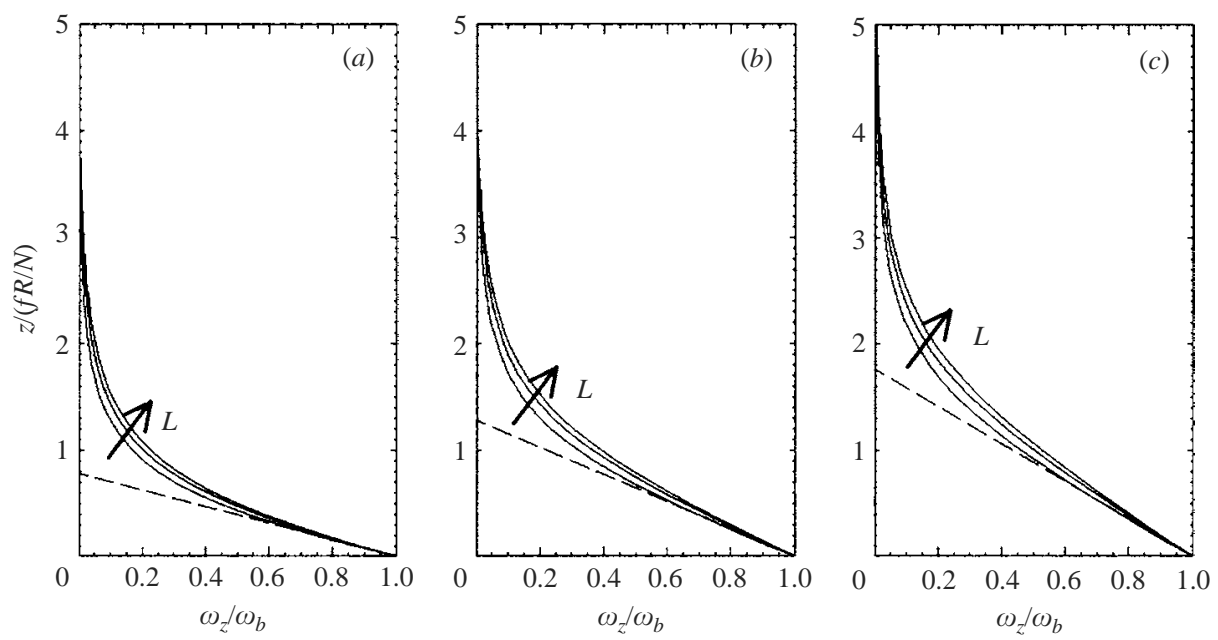

FiguRE 6. Profiles of non-dimensional vertical vorticity $\omega_{z} / \omega_{b}$ above the centre of the rotating disk, predicted by the stretching model for different values of the Rossby number $R o_{b}$. The vertical coordinate has been scaled with $f R / N$. Results from the stretching model for $L=0.5,1.0,1.5$ are represented by solid lines; the arrow indicates increasing $L$. $(a) R o_{b}=0$, (b) $R o_{b}=1.0$ and (c) $R o_{b}=2.0$. Dashed lines indicate the slope used to calculate the vertical extent, $H$.

impact of $L$ on the vertical size $H$ (discussed in the Appendix) is weak, the dependence of $H$ on $R o_{b}$ will be estimated using the results for $L=1.0$, and yields

$$
H_{(L=1.0)}=\frac{f R}{N}\left(0.8+0.6 R o_{b}\right) .
$$

\subsubsection{Evolution with time of the flow}

To predict the evolution of the flow with time, the numerical solutions for axisymmetric stationary flows in region III, described above, must be coupled to the Ekman circulation driven by region I.

For each stationary flow solution, the maximal displacement of isodensity surfaces is found at the centre of the disk. Integration of (4.1) along the vertical direction for $r=0$, using the fact that $\tilde{\rho}$ vanishes at infinite $z$, gives the value of the density perturbation at the centre of the disk, which in non-dimensional units is

$$
\tilde{\rho}\left(r=0, z=0, R o_{b}\right)=-\int_{0}^{\infty} \frac{\frac{1}{2} \omega(z, r=0)}{1+\frac{1}{2} R o_{b} \omega(z, r=0)} \mathrm{d} z,
$$

where $\omega(z, r=0)$ is the non-dimensional vertical vorticity along the vertical axis located above the disk centre. This density perturbation is associated with a vertical displacement, $d$, of the density surface crossing the centre of the disk. It is straightforward to show that $d$, in non-dimensional units, is

$$
d\left(R o_{b}\right)=R o_{b} \int_{0}^{\infty} \frac{\frac{1}{2} \omega(z, r=0)}{1+\frac{1}{2} R o_{b} \omega(z, r=0)} \mathrm{d} z,
$$

where $z$ is non-dimensionalized with $f R / N$. Values of $d$ can be easily calculated using the numerical solutions of (4.4).

During the spin-up, the displacement $d$ of the density surface at the bottom of region III is driven by the vertical velocity at the top of the boundary layer. To take 
account of the finite value of the Rossby number, results for a nonlinear boundary layer on a flat plate (see e.g. Greenspan 1968) are considered and fitted by the law $w_{\text {Ekman }}=\sqrt{\nu / \Omega_{w}}\left(\Omega_{w}-\Omega_{f}\right)$, with $\Omega_{w}$ and $\Omega_{f}$ the rotation speeds of the wall and the fluid outside the boundary layer, respectively. With our notation, $\Omega_{w}=\left(f+\omega_{d}\right) / 2$ and $\Omega_{f}=2\left(f+\omega_{b}\right) / 2$, so that (in dimensional units)

$$
\frac{f R}{N} \frac{\partial d}{\partial t}=w_{\text {Ekman }}=\sqrt{\frac{v}{2 f(1+R o)}}\left(\omega_{d}-\omega_{b}\right)
$$

where $v$ is the kinematic viscosity and $R o=\omega_{d} / f$ is the value of the Rossby number based on the vorticity of the rotating disk.

The non-dimensional vorticity at the base of region III can be written as $R o_{b}=$ $\alpha(t) R o$, where $\alpha(t)$ is a time-dependent function with $\alpha(t=0)=0$ and $\alpha(t=\infty)=1$. The equation governing the evolution of $\alpha$ with time can be deduced from (4.8):

$$
\frac{f R}{N} \frac{\partial}{\partial t}(d(\alpha(t) R o))=\sqrt{\frac{v}{2 f(1+R o)}}(1-\alpha(t)) \omega_{d} .
$$

For low values of $R o_{b}$, the expression for the function $d\left(R o_{b}\right)$ can be approximated by $d\left(R o_{b}\right) \approx a(L) R o_{b}$, where $a(L)$ is a constant depending on the choice of the model $(a(L)$ extends from 0.55 to 0.66 for $L$ between 0.5 and 1.5 ). The analytical solution of (4.9) for $R o \rightarrow 0$ is then

$$
\alpha(t)=1.0-\exp \left(-\frac{N}{a(L) R} \sqrt{\frac{\nu}{2 f}} t\right)
$$

which, after taking an average value for the constant $a(L)$ yields a typical spin-up timescale

$$
\tau_{s u}=0.6 \sqrt{\frac{2 f}{v}} \frac{R}{N}=1.2 E_{k}^{-1 / 2} N^{-1},
$$

with $E_{k}$ the Ekman number, $E_{k}=v / \Omega R^{2}$, where $\Omega=f / 2$. The latter definition of the spin-up time is in accordance with studies on the linear spin-up of stratified fluids (see e.g. Spence et al. 1992) and shows the similarity between the local spin-up in the neigbourhood of a small rotating disk and the spin-up in cylindrical tanks. Indeed, the main effect of stratification is to confine the flow to a height $H=f R / N$, with $R$ the horizontal extent of the forcing, and an homogenous fluid in a tank of height $H$ is known to be spun-up by the Ekman boundary circulation on a timescale given by $E^{-1 / 2} \Omega^{-1}$, with $E=v / \Omega H^{2}$. Combination of these two features for a rotating disk flow leads to the same scaling as in (4.11).

For large values of $R o_{b}$, the function $d\left(R o_{b}\right)$ cannot be approximated by a linear relationship. Numerical solutions of (4.4) are used in (4.7) to determine how $d\left(R o_{b}\right)$ evolves with $R o_{b}$. Equation (4.9) becomes nonlinear and has to be solved numerically. These results will be presented in the next section (for the stretching model in figures 8 , 10, 11 and 12).

For completeness, we estimate the importance of the time-dependent term $\partial(\partial u / \partial z-$ $\partial w / \partial r) / \partial t$ which appears in the fully time-dependent form of the thermal wind equation (4.2), with $u$ and $w$ respectively the radial and vertical components of the velocity. This term was neglected in the present approach. The vertical velocity $w(t)=\omega_{d} \sqrt{\nu / f}(1-\alpha(t))$, which is maximal at the bottom of region III, decreases with time. This vertical flux is balanced by a radial transport distributed along the vertical height $H=0.8 f R / N$ of the vortex. By continuity the radial velocity $u(t)$ above the 
disk edge satisfies $u(t) \approx w(t) N / f$. Therefore,

$$
\frac{\partial u}{\partial z}-\frac{\partial w}{\partial r} \approx\left((N / f)^{2}-1\right) w / R
$$

yielding

$$
\partial(\partial u / \partial z-\partial w / \partial r) / \partial t \approx\left((N / f)^{2}-1\right) \sqrt{v / f}\left(\omega_{d} / R\right) \partial \alpha(t) / \partial t,
$$

to be compared to the Coriolis term

$$
f(\partial v / \partial z) \approx f \omega_{d}(N / f) \alpha(t) .
$$

Since $(\partial \alpha / \partial t) / \alpha=1 / \tau_{s u}$ and $N / f>1$, the time-dependent term can be neglected as long as $(N / f R) \sqrt{\nu / f}\left(f \tau_{s u}\right)^{-1} \ll 1$. This condition can also be written $(\delta / H)\left(f \tau_{s u}\right)^{-1} \ll 1$, with $\delta$ the Ekman layer thickness and $H$ the vertical extent of the vortex. In our experiments, the maximal value of $\left(f \tau_{s u}\right)^{-1}$ was around 0.5 , but in most cases, this value remained under 0.3 , and $(\delta / H)$ was smaller than 1 , so that the time-dependent terms can be neglected in the description of the flow in region III.

\subsection{Diffusive model}

For certain experimental parameters the vertical extent of the vortex predicted by the stretching model, $H$, was initially small, leading thus to a relatively faster transport of momentum by diffusion than by the inviscid spin-up. Therefore, we assume a purely diffusive evolution from rest of the flow in region III. Besides, on times far larger than the inviscid spin-up time $\tau_{s u}$, diffusive transport of momentum may also overcome the momentum generated by the vortex stretching. In the present approach, the rotating disk will be considered as a source of momentum and all other effects, such as stretching of background vorticity and radial or vertical movements, will be neglected.

The evolution of the azimuthal velocity $v(r, z, t)$ can then be described by a twodimensional diffusion equation (see e.g. Clercx et al. 2001). Using the scaling $(r, z, t) \rightarrow$ $\left(R r, R z, R^{2} / v\right)$ and $v \rightarrow\left(\omega_{d} R / 2\right) v$, where $\omega_{d}$ is the vorticity of the body rotation of the disk, $R$ is the disk radius and $v$ is the kinematic viscosity, it takes the following non-dimensional form:

$$
\frac{\partial v}{\partial t}=\frac{\partial^{2} v}{\partial^{2} r}+\frac{\partial v}{r \partial r}-\frac{v}{r^{2}}+\frac{\partial^{2} v}{\partial z^{2}} .
$$

At $t=0$, the disk starts to rotate in a fluid at rest, so that the boundary conditions are

$$
\left.\begin{array}{lll}
t=0, & r<1: & v(r, 0,0)=r, \\
& \text { elsewhere }: & v(r, z, 0)=0, \\
t>0, & r<1: & v(r, 0, t)=r .
\end{array}\right\}
$$

This problem was solved numerically by a Runge-Kutta algorithm of fourth order applied to equation (4.12) in a domain surrounding the rotating disk. During the numerical integration, the velocity on the disk tends to decrease with time, as expected for a free diffusive evolution. Therefore, to fulfil the boundary conditions (4.13) for $t>0$, the profile of vorticity on the disk surface was maintained constant at each step of the integration and very short time-steps were chosen in order to limit the numerical noise.

In figure 7 , the evolution of the vertical vorticity profile above the centre of the disk is plotted, next to the solution for an infinite rotating disk, for which equation (4.12) simplifies into a one-dimensional diffusive equation and yields solutions of the form $\omega(z, t)=\omega_{d} \operatorname{erfc}(-z / 2 \sqrt{t})$. Initially, the two evolutions are exactly the same, but 


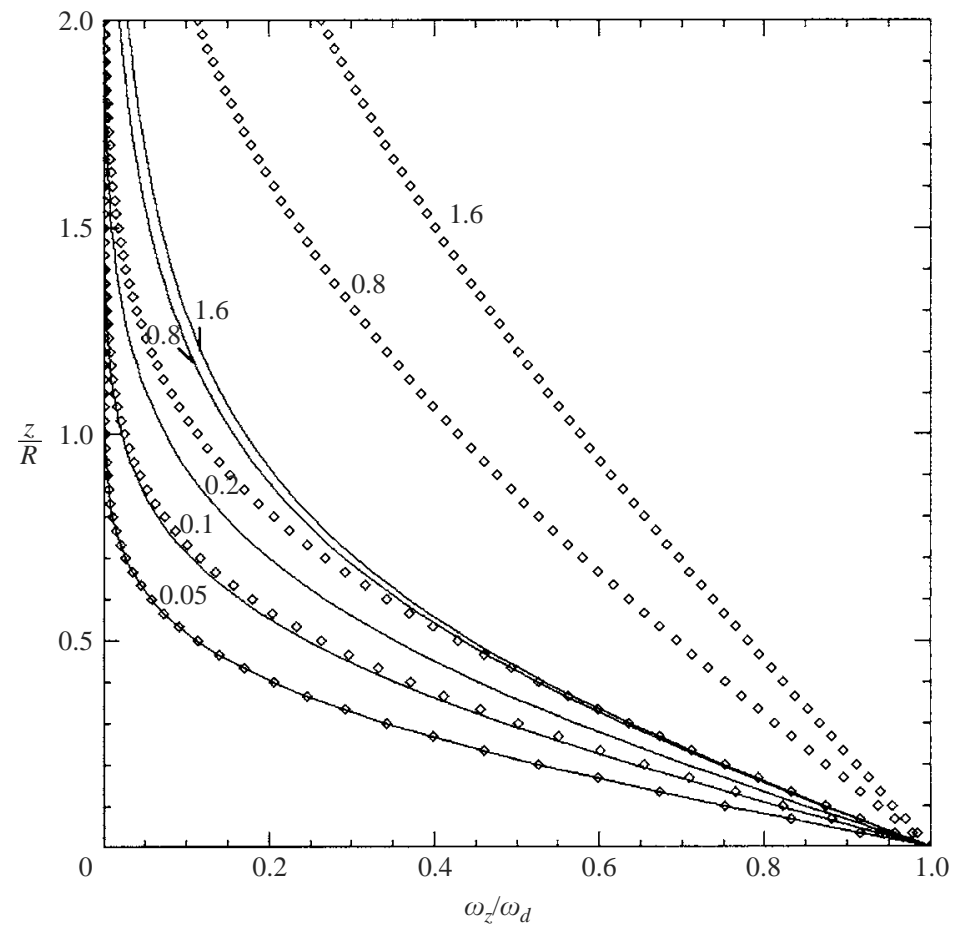

FIGURE 7. Evolution of the profile of non-dimensional vertical vorticity $\omega_{z} / \omega_{d}$ above the centre of the rotating disk, predicted by the diffusive model. The vertical coordinate, $z$, is scaled with the disk radius $R$. Solid lines represent the predictions by the diffusive model for non-dimensional times, $t /\left(R^{2} / v\right)=0.05,0.1,0.2,0.8$ and 1.6 respectively, as labelled; the diamonds represent the results of the corresponding one-dimensional analytical solution for the same times.

around a non-dimensional time 0.2 , when the vertical and horizontal extents of the flow induced by the finite rotating disk are similar, radial diffusion slows down the relative growth of $\omega(z)$ (see figure 7). The dimensional value $\tau_{v}$ of this time is given by

$$
\tau_{v}=0.2 \frac{R^{2}}{v} .
$$

For $t>\tau_{v}$, the further evolution becomes similar to the problem of a point source of momentum located at the disk centre. For $t<\tau_{v}$, a one-dimensional analytical expression for the diffusive problem can be used to estimate the vertical extent of the vortex as a function of time. Using the same definition as for the stretching model, based on the slope of the vorticity profile above the centre of the disk, the evolution of the vertical extent of the vortex follows

$$
H_{v}(t)=\sqrt{\pi v t}
$$

For $t=\tau_{v}$, the vertical extent as defined here equals $0.8 R$.

\subsection{Modelling of instabilities}

\subsubsection{Instability of the density front}

The continuous release of a light fluid by the Ekman layer generates an axisymmetric gravity current which spreads until it becomes unstable to non-axisymmetric 
disturbances. The dynamics of this gravity current generated by Ekman pumping is not dissimilar to the axisymmetric gravity current produced by the annular source flow described in Griffith \& Linden (1981) or, in the unidirectional case, the finite release of a line plume in a stratified fluid in Bush \& Woods (1999).

In Griffith \& Linden (1981), the axisymmetric gravity current is generated by the continuous release of fluid from an annular source in a rotating tank of height $H$ containing a homogenous relatively dense fluid. As long as the height $h$ of the current is a significant fraction of the local depth, the instability is well-described by baroclinic instability of a two-layer fluid in a rotating channel of rectangular cross-section; the dimensions of the cross-section are prescribed by the shape of the gravity current. The instability is driven by the vertical shear and its wavelength scales as several times the radius of deformation, $\sqrt{g^{\prime} h} / f$, where $g^{\prime}$ is the reduced gravity. In our experiments, the fluid is linearly stratified, so that Eady's model (see Eady 1949) for baroclinic instability is more appropriate. When the height, $H_{f}$, and the width, $L_{f}$, of the channel are prescribed by the height and the width of the density front, respectively, the flow should become baroclinically unstable for values of the Burger number, $B u=\left(N H_{f} / f L_{f}\right)^{2}$, below a critical value $B u_{c}=0.6$, with a wavelength $\lambda_{b c} \approx 4 N H_{f} / f$ for the most unstable mode. This wavelength scale is several times the radius of deformation for a stratified fluid.

Bush \& Woods (1999) show how a finite release of a line plume in a rotating stratified fluid generates a density current which forms and spreads radially at its level of neutral buoyancy and when unstable breaks up into an array of anticyclonic vortices. The subsequent flow cascade to larger scales is very similar to what we observe (see figure 4). The number of vortices can be modelled by supposing continuity of mass between the line plume flux and the final array of vortices, and using a constant Burger number $B u=\left(N R_{v} / f H_{v}\right)^{2}$ for the emerging vortices by Bush \& Woods (1999), where $R_{v}$ and $H_{v}$ are the radius and height of the vortices. The constraint on the Burger number of emerging vortices is consistent with the fully nonlinear development of an instability whose wavelength would scale as several times the radius of deformation of the gravity current, showing the link between the instability studied by Bush \& Woods (1999) and the baroclinic instability mentioned above. There exists an upper limit for the value of a vortex Burger number, since high values are associated with a vortex radius far larger than the Rossby radius of deformation, and such vortices are then baroclinically unstable, splitting into smaller vortices. In other words, the vortices emerging from the unstable density front adopt values of $B u$ compatible with their stability, and are prescribed by baroclinic instability of the initially axisymmetric density current.

In our experiments, the volume flux of fluid ejected at the edge of the rotating disk during the spin-up becomes a function of time, and is limited by the volume prescribed by the vertical extent of the vortex at the end of the spin-up, as predicted by the stretching model. This final state is achieved on the timescale $\tau_{s u}$, defined in (4.11). Following the approach proposed by Bush \& Woods (1999), the volume per unit length, $V(t)$, available to generate vortices at time $t$, scales roughly as

$$
V(t) \sim(f R / N) d(R o) R\left(1-\exp \left(-t / \tau_{s u}\right)\right),
$$

where $R$ is the disk radius, $(f R / N) d(R o)$ the vertical displacement of the density surface crossing the centre of the disk at the end of the spin-up, and $d(R o)$ is given by (4.7). Continuity requires that $V(t) \sim R_{v}(t) H_{v}(t)$ where $R_{v}(t)$ and $H_{v}(t)$ are the radius and height of the vortices that would emerge from the instability at time $t$. Assuming a constant Burger number for the vortices, as suggested by Bush \& Woods (1999), we obtain $R_{v} \sim N H_{v} / f$, and deduce $R_{v}$ as a function of time from the continuity 


\begin{tabular}{cccccccccc}
\hline Expt & $f\left(\mathrm{~s}^{-1}\right)$ & $N\left(\mathrm{~s}^{-1}\right)$ & $R(\mathrm{~cm})$ & $\omega_{d}\left(\mathrm{~s}^{-1}\right)$ & $t(\mathrm{~min})$ & $R o$ & $N / f$ & $R e$ & $\left(\tau_{\text {su }} f\right)^{-1}$ \\
$a$ & 0.50 & 1.19 & 6.0 & 0.31 & 29 & 0.63 & 2.38 & 560 & 0.07 \\
$b$ & 0.50 & 1.19 & 6.0 & 0.31 & 42 & 0.63 & 2.38 & 560 & 0.07 \\
$c$ & 0.13 & 1.17 & 6.0 & 0.31 & 28 & 2.49 & 9.29 & 560 & 0.50 \\
$d$ & 0.18 & 1.16 & 6.0 & 0.18 & 24 & 1.00 & 6.45 & 320 & 0.30 \\
$e$ & 0.18 & 1.16 & 6.0 & 0.60 & 13 & 3.33 & 6.45 & 1080 & 0.30 \\
$f$ & 0.31 & 1.16 & 6.0 & 0.18 & 20 & 0.57 & 3.69 & 320 & 0.13 \\
$g$ & 0.38 & 1.16 & 6.0 & 0.31 & 21 & 0.84 & 3.09 & 560 & 0.10 \\
$h$ & 0.63 & 1.45 & 6.0 & 0.18 & 18 & 0.29 & 2.32 & 320 & 0.06 \\
$i$ & 0.63 & 1.45 & 6.0 & 0.31 & 20 & 0.50 & 2.32 & 560 & 0.06 \\
$j$ & 0.63 & 1.45 & 6.0 & 0.47 & 18 & 0.74 & 2.32 & 850 & 0.06 \\
$k$ & 0.31 & 1.45 & 6.0 & 0.47 & 16 & 1.49 & 4.64 & 850 & 0.17 \\
$l$ & 0.31 & 1.45 & 6.0 & 0.31 & 15 & 1.00 & 4.64 & 560 & 0.17 \\
$A$ & 0.26 & 1.4 & 12.0 & 0.13 & 40 & 0.52 & 5.47 & 940 & 0.10 \\
$B$ & 0.26 & 1.4 & 12.0 & 0.24 & 44 & 0.93 & 5.47 & 1730 & 0.10 \\
$C$ & 0.26 & 1.4 & 12.0 & 0.38 & 33 & 1.49 & 5.47 & 2700 & 0.10 \\
$D$ & 0.26 & 1.4 & 12.0 & 0.52 & 31 & 2.05 & 5.47 & 3700 & 0.10 \\
$E$ & 0.38 & 1.4 & 12.0 & 0.13 & 18 & 0.35 & 3.67 & 940 & 0.06 \\
$F$ & 0.38 & 1.4 & 12.0 & 0.38 & 35 & 1.00 & 3.67 & 2700 & 0.06 \\
& & & & & & & &
\end{tabular}

TABLE 1. Values of experimental parameters for stable vortices generated by a rotating disk. The Rossby number $R o$ is defined as $R o=\omega_{d} / f$. The Reynolds number $R e$ is based upon the radius of the rotating disk, $R e=\omega_{d} R^{2} / \nu . \tau_{s u}$ is the spin-up time defined in (4.11).

relationship. The number of vortices is thus given by $n \sim 2 \pi R / R_{v}(t)$, or

$$
n(t)=C\left[d(R o)\left(1-\exp \left(-t / \tau_{s u}\right)\right)\right]^{-1 / 2},
$$

with $C$ a constant of proportionality.

\subsubsection{Formation of layers in region II}

On very large times $\left(t \gg \tau_{s u}, \tau_{d i f f}\right)$, layers appear in region II, above the edge of the disk, and are detected with the shadowgraph method. These layers have been studied experimentally by Baker (1971) and are generated in flows where local values of the Richardson number $R i=N^{2} /(\partial u / \partial z)^{2}$ are below 175. Different values for the salt diffusivity $\kappa$, and the kinematic viscosity $\nu$, are required and lead to overturning motions only in ring-shaped regions for a vortex flow (see Calman 1977). In our experiments, this instability develops only above the edge of the rotating disk, on times larger than $\tau_{s u}$ and $\tau_{d i f f}$, and is therefore neglected.

\section{Comparison with experimental results}

Experiments were carried out with two values of the disk radius, $R=6 \mathrm{~cm}$ and $R=12 \mathrm{~cm}$, and various values of the relevant experimental parameters $\omega_{d}, f$ and $N$, during the initial spin-up of fluid around the rotating disk.

PIV measurements in a horizontal plane (typically $2 \mathrm{~cm}$ above the disk) provide information about the evolution of the vertical vorticity. After the development of the instability of the density front and once the vortex attained a quasi-stationary axisymmetric state, PIV measurements were carried out in horizontal planes at different heights above the disk in order to obtain a view of the fully three-dimensional structure of the vortex. Values of the experimental parameters as well as the relevant non-dimensional numbers introduced in the previous sections are given in table 1 for various experiments. 

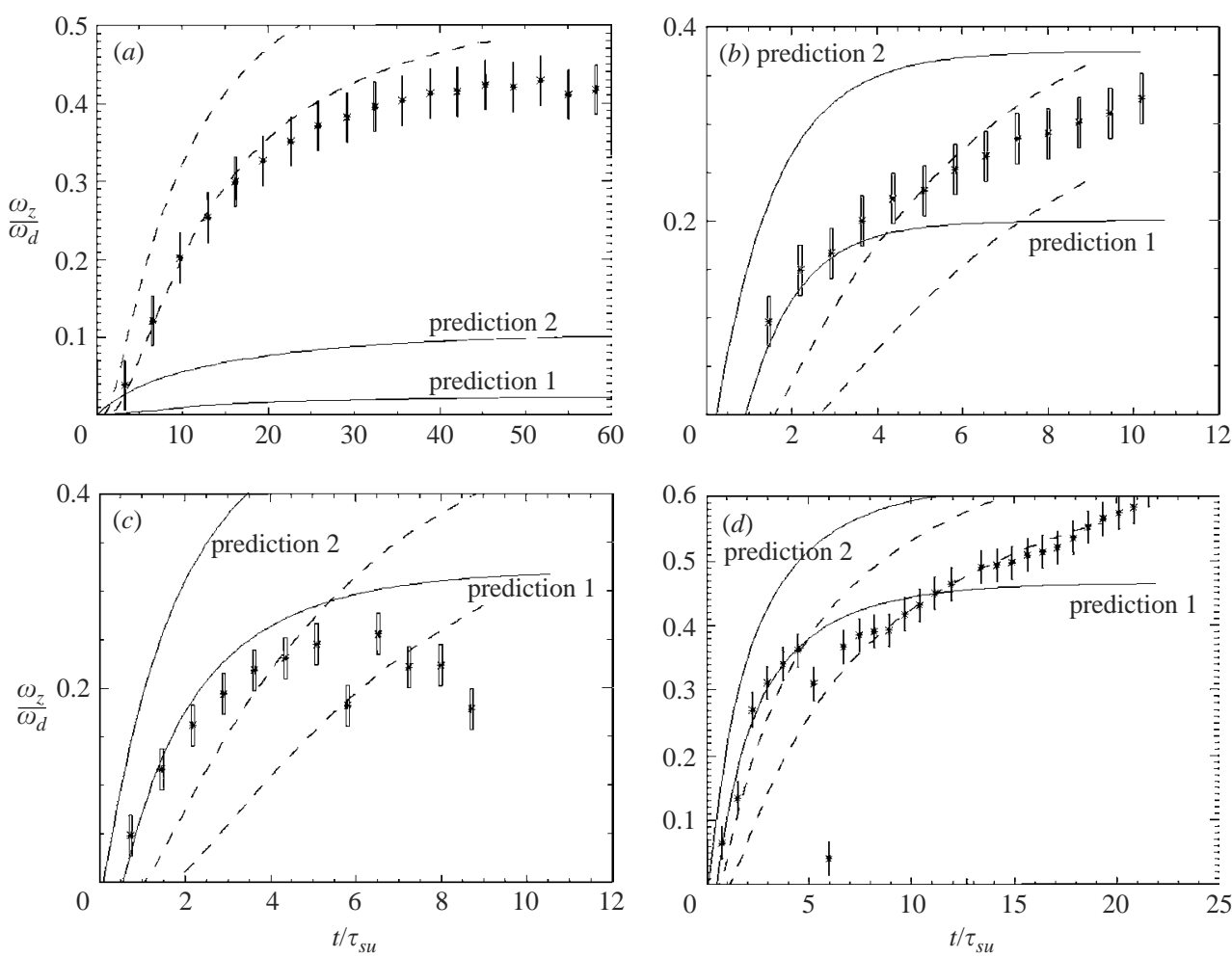

FIGURE 8 . Evolution of the vertical vorticity $\omega_{z} 2 \mathrm{~cm}$ above the centre of the rotating disk for experiments $(a) c,(b) h,(c) j$ and $(d) F$ (see tables 1 and 2 for experimental parameters). Dashed lines: the diffusive model for heights $1.75 \mathrm{~cm}$ and $2.25 \mathrm{~cm}$; solid line labelled prediction $1:$ the stretching model for $L=0.5$, height $2.25 \mathrm{~cm}$ and a delay for the initial time $(\sim 4 \pi /(f+w d))$ in order to reproduce the formation of the Ekman layer; solid line labelled prediction 2: the stretching model for $L=1.5$, height $1.75 \mathrm{~cm}$, without delay. *, experimental values based upon PIV measurements.

In the next subsection, we will compare measurements obtained for the initial axisymmetric evolution just after the onset of the disk rotation with predictions by the stretching model and the diffusive model. Then, we will focus on the density front instability, and finally we will consider measurements and predictions for the final axisymmetric state.

\subsection{Initial spin-up, $t \geqslant 0$}

Figure 8 shows measurements of the maximum vertical vorticity in a layer $2 \mathrm{~cm}$ above the rotating disk and predictions from the stretching and diffusive models as a function of time. For large $N / f$ (see figure $8 a$ ), on a timescale given by $\tau_{s u}$, the stretching model predicts a very small increase of the central vorticity in the region above the rotating disk. On the same timescale, the diffusive model predicts a larger increase of vorticity and reproduces the experimental measurements very well. For small $N / f$ (see figure $8 b$ ), the initial growth of vorticity is predicted well by the stretching model, but underestimated by the diffusive model, thus showing the dominant effect of vortex stretching by Ekman pumping.

To distinguish these two mechanisms, we compare the diffusive timescale with the inviscid spin-up time. The diffusive timescale, $\tau_{\text {diff }}$, when based on the vertical extent 


$\begin{array}{ccccrc}\text { Expt } & R o & N / f & \tau_{s u}(\mathrm{~s}) & \tau_{v}(\mathrm{~s}) & \gamma \\ c & 2.49 & 9.29 & 16 & 720 & 0.5 \\ h & 0.29 & 2.32 & 28 & 720 & 4.8 \\ j & 0.74 & 2.32 & 28 & 720 & 4.8 \\ F & 1.00 & 3.67 & 45 & 2880 & 4.75\end{array}$

TABLE 2. Experimental results for the study of the spin-up just at the onset of the disk rotation. Definitions of $\tau_{s u}, \tau_{v}$ and $\gamma$ are given respectively in (4.11), (4.14) and (5.2).

of the vortex $H$ predicted by the stretching model with (4.15) is given by

$$
\tau_{\text {diff }}=\left(\frac{0.8 f R}{N}\right)^{2} \frac{1}{\pi v},
$$

and represents the time required for diffusive processes to generate the same thickness as obtained by stretching of background vorticity in time $\tau_{s u}$. Its ratio with the spin-up time $\tau_{s u}$, denoted $\gamma$, is

$$
\gamma=\frac{\tau_{\text {diff }}}{\tau_{s u}} \approx\left(\frac{f}{N}\right)^{2} \frac{\tau_{v}}{\tau_{s u}} .
$$

In table 2, values of this parameter are given for the experiments presented in figure 8 .

As shown by Walin (1969) in the framework of the linear theory $(R o \rightarrow 0)$, the penetration depth for the secondary circulation is scaled by the Prandtl depth, $D=$ $f R / N$, and the Ekman layer thickness is $\delta=\sqrt{2 v / f}$. Using these lengthscales, $\gamma=D / 6 \delta$ (or $\gamma=H / 5 \delta$ if we use results from the stretching model). Therefore, in the linear approximation, $\gamma \ll 1$ indicates that the vertical scale for the secondary circulation is smaller than the Ekman boundary layer thickness, and this secondary circulation is then shut down very quickly. For $\gamma \approx 1$, lengthscales are different, but timescales are identical, indicating the limit between the two dominant processes driving the spin-up.

For nonlinear spin-up $(R o \neq 0), \gamma$ is more conveniently defined as a ratio of timescales for these two processes, and values calculated within the low-Ro limit are used to classify the experiments. For $\gamma<1$, the spin-up is due to diffusion and the evolution of vorticity can be predicted by diffusion alone, as shown in figure $8(a)$. For $\gamma>1$, momentum transport by stretching initially dominates (see figures $8 b, c$ ), whereas in a second stage, for $t>\tau_{s u}$, the evolution is driven by diffusive processes. This diffusive evolution appears clearly in the right part of figures $8(b)$ and $8(d)$, where the approximately constant increase of vorticity at large times corresponds to the diffusive evolution. It should be noted that the transition in figure $8(d)$ to this second stage coincided with the transition to the non-axisymmetric transient stage associated with the instability of the density front.

\subsection{Instability of the density front}

In order to determine the nature of this instability, experiments with the large disk $(R=12 \mathrm{~cm})$ were carried out for different values of the experimental parameters. The vertical and radial extents $H_{f}$ and $L_{f}$ of the front were measured from flow visualizations in a vertical plane with fluorescein dye released on the disk surface (see figure 2) or solder particles released close to the edge of the disk. The horizontal wavelength of the instability was measured by illuminating the dye or the solder particles with a horizontal laser sheet as in figure 4 . 


\begin{tabular}{|c|c|c|c|c|c|c|c|c|c|}
\hline Expt & $\omega_{d}\left(\mathrm{~s}^{-1}\right)$ & $f\left(\mathrm{~s}^{-1}\right)$ & $N\left(\mathrm{~s}^{-1}\right)$ & $H_{f}(\mathrm{~cm})$ & $L_{f}(\mathrm{~cm})$ & $t_{\text {mes }}(\mathrm{s})$ & $B u$ & $\lambda_{\exp }(\mathrm{cm})$ & $n(t)$ \\
\hline 1 & 0.6 & 0.6 & 1.8 & 1.5 & 4.0 & $\begin{array}{r}60 \\
95 \\
150\end{array}$ & 1.3 & 15.0 & $\begin{array}{l}5 \\
4 \\
3\end{array}$ \\
\hline 2 & 0.6 & 0.6 & 1.8 & 1.5 & 3.2 & 52 & 2.0 & 15.0 & 5 \\
\hline 3 & 0.3 & 0.6 & 1.5 & 1.0 & 2.0 & $\begin{array}{r}39 \\
66 \\
112 \\
164\end{array}$ & $\begin{array}{l}1.6 \\
1.6 \\
1.6 \\
1.6\end{array}$ & 9.0 & $\begin{array}{l}9 \\
8 \\
6 \\
3.5\end{array}$ \\
\hline 4 & 0.3 & 0.7 & 1.8 & 1.0 & 2.7 & 60 & 0.9 & 10.0 & 8 \\
\hline 5 & 0.6 & 0.7 & 1.8 & 1.5 & 1.8 & 60 & 2.1 & 12.0 & 6 \\
\hline 6 & 0.3 & 0.5 & 1.3 & 1.0 & 1.8 & 80 & 2.1 & 15.0 & 5 \\
\hline 7 & 0.2 & 0.5 & 1.3 & 1.0 & 2.8 & 250 & 0.9 & 19.0 & 4 \\
\hline
\end{tabular}

TABLE 3. Measurements of density front extents and instability wavelength for different experiments with the large rotating disk $R=12 \mathrm{~cm} . \omega_{d}$ : disk vorticity, $f$ : Coriolis parameter, $N$ : buoyancy frequency, $H_{f}$ and $L_{f}$ : vertical and horizontal extents of the density front measured in a vertical plane when the density front instability becomes visible. $B u$ : estimation of the value for the Burger number $B u=\left(N H_{f} / f L_{f}\right)^{2}$ at the onset of the instability, $t_{m e s}$ : time after the onset of the disk rotation, for which the instability wavelength $\lambda_{\exp }$, or the number of surrounding vortices $n\left(t_{m e s}\right)$, are measured in an horizontal plane.

During the growth of the density front, the values of the Burger number based on measurements of the radial and vertical extents $H_{f}$ and $L_{f}$ of the front, $B u=$ $\left(N H_{f} / f L_{f}\right)^{2}$, were found to be high initially, and decreased with time as the front spread out radially. The values of this Burger number when the instability becomes visible in vertical flow visualizations are given in table 3 . For the different experiments, the order of magnitude is the same as the critical value, $B u_{c}=0.6$, required to give rise to a baroclinic instability in the Eady model (see Eady 1949). The wavelength of this first instability was measured from dye visualizations in a horizontal plane close to the rotating disk, whereas the front height was measured from separate experiments for the same initial experimental parameters. In particular the measurement of the front height $H_{f}$ was sensitive to the initial dye distribution. In order to compare the experimental results with the theorical predictions for the baroclinic instability model of Eady, measurements of the instability wavelength are represented as a function of $N H_{f} / f$ in figure $9(a)$. The experimental measurements are not very well reproduced by Eady's model based on the shape of the density front, mainly because of large errors in the measurements of $H_{f}$ at the onset of the instability. Nevertheless, the model gives a reasonable estimation of the wavelength, suggesting that the mechanism for the instability is baroclinic.

Wave-like irregularities begin to occur $30 \mathrm{~s}$ after the onset of the disk rotation as shown in figure 4 and in agreement with the observations of Bush \& Woods (1999) for the same background rotation rate. In contrast with the predictions based on the Eady model that are relevant only for the first instability, the model proposed by Bush \& Woods (1999) explains the decreasing number of vortices observed in the experiments as time goes on. We used horizontal flow visualisation to measure the number of vortices, $n$, at successive times $t_{m e s}$. In order to compare the experimental results with the prediction inferred from the model of Bush \& Woods (1999), measurements of $n$ were represented as a function of the right-hand side of (4.16) in figure $9(b)$. For $t_{m e s}<\tau_{s u}$ (all with $n>4$ ) good agreement is found between experimental results and the model predictions, thus confirming that baroclinic instability is the dominant 

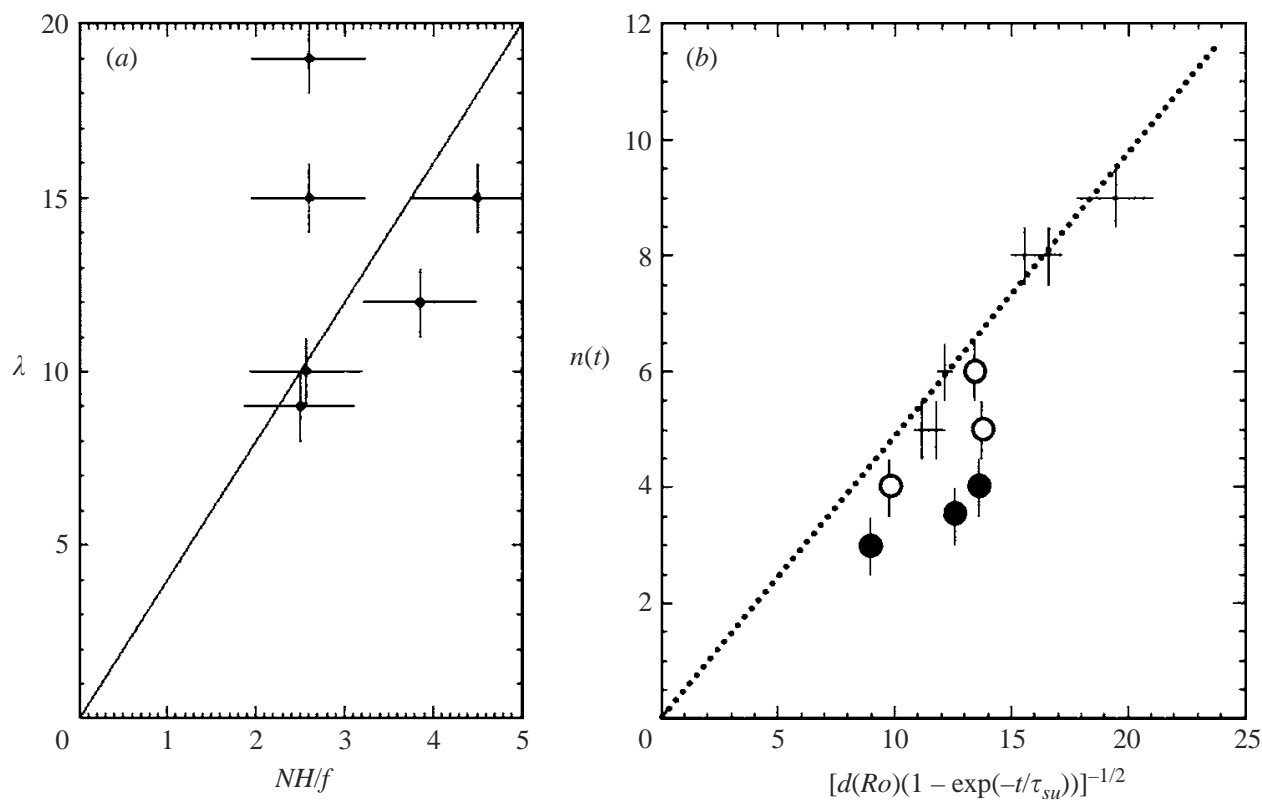

FIGURE 9. Comparison of the density front instability pattern observed in the experiments and the predictions from the models presented in $\S 4.3$. (a) Comparison of the wavelength of the first instability with prediction from the Eady model. The solid line corresponds to $\lambda=4 N H_{f} / f$ and the error bars are determined by the standard deviation in the density front vertical extent $H_{f}$. (b) Comparison of the number of surrounding vortices with prediction from (4.16). Open and solid circles indicate measurements at $t \in\left[\tau_{s u}, 2 \tau_{s u}\right]$ and $t>2 \tau_{s u}$, respectively. Horizontal error bars are based on a delay in the formation of the Ekman boundary layer $(\sim 4 \pi /(f+w d))$. See table 3 for experimental parameters.

mechanism. A growing discrepancy is observed as $t_{m e s}$ increases beyond $\tau_{s u}, n$ continuing to decrease with time when the density front is no longer fed. The latter behaviour is consistent with the fact that multipolar vortices with $n \geqslant 4$ are unstable and evolve towards low-order multipolar vortices $(n \leqslant 3)$ through successive merging of the surrounding vortex cores. In our experiments, the flow cascade to larger scales is predicted and explained well by the model of Bush \& Woods (1999) for $t_{m e s} \leqslant \tau_{s u}$, whereas for $t_{m e s}>\tau_{s u}$, interactions between the anticyclonic vortices and/or with the vortex cyclonic core become the driving mechanism.

Note that for values of $n$ below 4, the instability pattern begins to affect the flow away from the density front (region III in our models presented in $\S 4$, and of figure $3 f$ ), but only for times larger than $\tau_{s u}$. As a consequence, the effect of the instability of the density front on the initial stage of the spin-up is weak and the modelling of flows in regions II and III remains valid. However, the transient through a non-axisymmetric stage with $n \leqslant 4$ in region III for $t \gg \tau_{s u}$ is most likely the source of discrepancy between the model predictions and the measurements for the final axisymmetric state of the vortex, i.e. the axisymmetric state towards which the flow eventually returns. This is discussed in the next subsection.

\subsection{Final axisymmetric state, $t>\tau_{\text {su }}, \tau_{\text {diff }}$}

Here, we consider the final axisymmetric state. In order to compare the experimental measurements and the model predictions, we use vertical and radial cross-sections of vertical vorticity, both measured from horizontal velocity fields. A relevant scaling 


$\begin{array}{cccccccc}\text { Expt } & t(\min ) & R o & N / f & \tau_{v}(\min ) & \gamma & t_{o b s} & t_{o b s}[R o] \\ c & 28 & 2.49 & 9.29 & 12 & 0.5 & 201 & 26 \\ B & 44 & 0.93 & 5.47 & 48 & 2.6 & 27 & 10 \\ h & 18 & 0.29 & 2.32 & 12 & 4.8 & 8.1 & 5.5 \\ E & 18 & 0.35 & 3.67 & 48 & 4.75 & 5.1 & 3.2\end{array}$

TABLE 4. Experimental parameters and non-dimensional observation times for the experiments of figures 10, 11 and 12. Definition of $\gamma$ is given in (5.2). $t_{o b s}$ is the non-dimensional time defined in (5.3), while $t_{o b s}[R o]$ takes account of the finite value of the Rossby number.

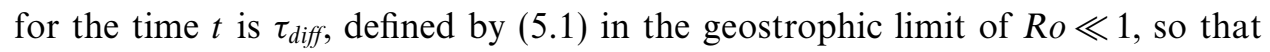

$$
t_{\text {obs }}=\frac{t}{\tau_{\text {diff }}} \approx\left(\frac{N}{f}\right)^{2} \frac{t}{\tau_{v}},
$$

and is an estimation of the amount of vorticity transported by diffusive processes in comparison with the vorticity generated by the stretching.

In the ageostrophic case, i.e. for finite values of the Rossby number Ro, one can use (4.6) to evaluate the vortex thickness $H$ obtained by stretching, and derive the non-dimensional observation time $t_{o b s}[R o]$. Values of the relevant non-dimensional numbers for the four typical experiments discussed below are given in table 4 .

\subsubsection{Vertical profiles of vertical vorticity above the center of the disk}

For the experiments presented in table 4, experimental profiles and predictions from the diffusive and stretching models are plotted in figure 10. For high values of $t_{o b s}[R o]$ (see table 4, experiments $c, B$ and figure $10(a)$ and $10(b)$ respectively), the diffusive model shows excellent agreement with experimental measurements. In these experiments, the observation time $t$ is larger than the diffusive time $\tau_{v}$, which separates the one-dimensional from the three-dimensional diffusive regime, and an analytical solution for the one-dimensional diffusive equation would over-estimate the experimental measurements.

For moderate values of $t_{o b s}[R o]$ (see table 4, experiments $h, E$ and figures $10(c)$ and $10(d)$, respectively), the experimental measurements are located between the curves predicted by the stretching model and the diffusive model. As the diffusion of momentum tends to decrease the vertical shear, the thermal wind balance expressed by (4.2) is broken and isodensity surfaces move back upward. This upward displacement was confirmed by experimental observations, and is associated with radial outflow and vortex compression that locally decreases the value of the vertical vorticity. By continuity, some inward transport occurs near the disk surface. This mechanism explains why the diffusive transport of momentum above the rotating disk is partially cancelled by the vortex compression effect, and the spin-up is slower than would be produced by diffusive processes alone. Such a meridional circulation was also discussed by Clercx et al. (2001) in their study on the spin-down of pancake-like vortices. They pointed out that this circulation is driven by diffusion and may generate a 'vortex stretching' effect in the mid-plane of the vortex, which leads to a slower spin-down than predicted by a purely diffusive model. In a related study by Flierl \& Mied (1985) on the diffusive spin-down of oceanic warm core rings and consequent meridional circulation, a similar slower decay for the baroclinic mode than for the barotropic mode was found. 

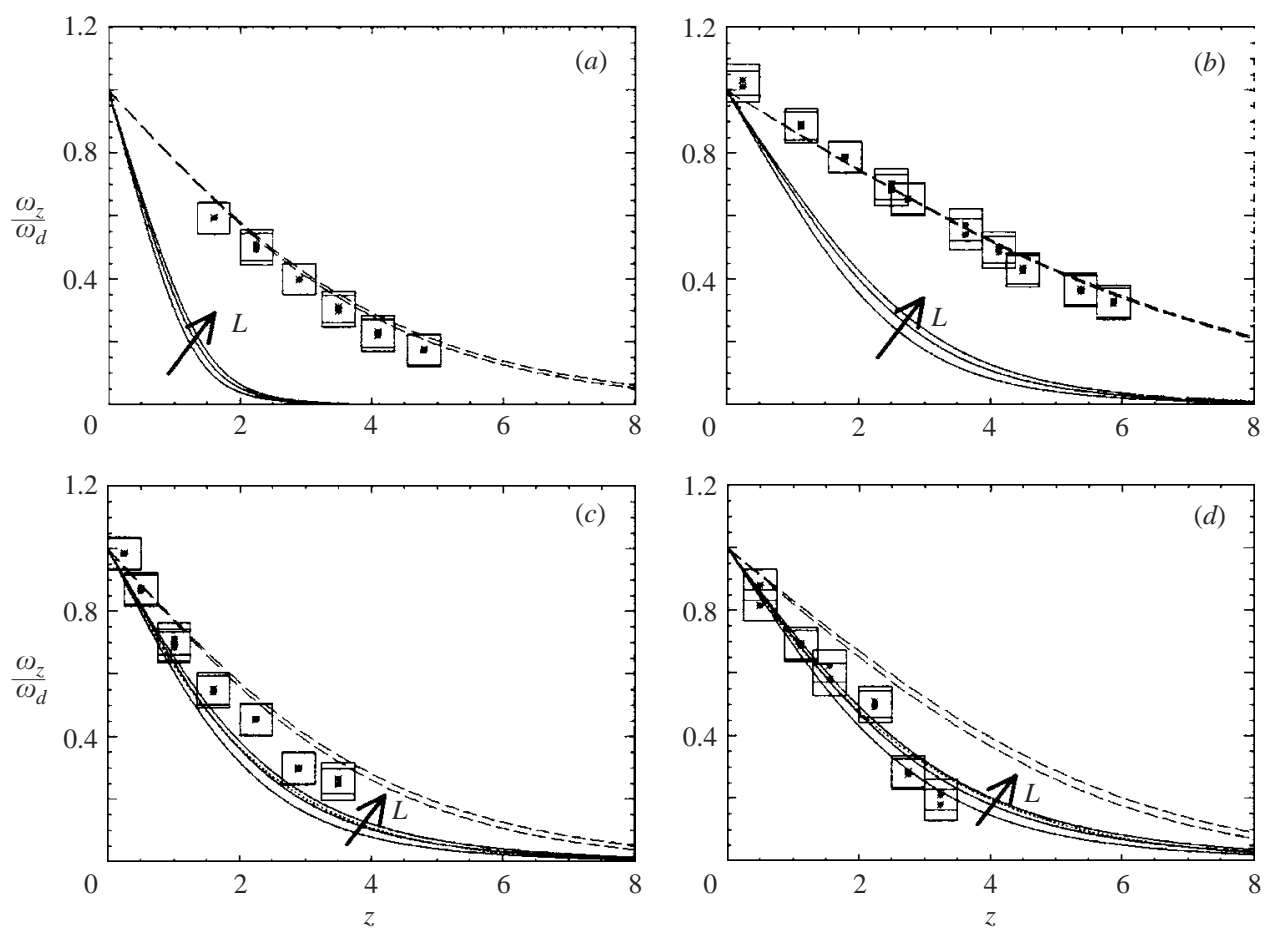

FIGURE 10. Vertical profiles of non-dimensional vertical vorticity $\omega_{z} / \omega_{d}$ above the centre of the rotating disk for experiments $(a) c,(b) B,(c) h$ and $(d) E$ : ----- , the diffusive model; the stretching model for $L=0.5, L=1.0$ and $L=1.5$. The arrow indicates increasing $L$ for the stretching models. An error of about $\pm 0.25 \mathrm{~cm}$ was found for the height of the horizontal laser sheet, and the vorticity has a precision of around $\pm 5 \%$. See table 4 for experimental parameters.

\subsubsection{Radial profiles of vorticity}

In figures 11 and 12, radial profiles of vertical vorticity at different heights above the rotating disk are plotted for two different experiments. The predictions by the diffusive model and the stretching model with $L=0.5$ are presented in figures $11(a)$, $12(a)$ and 11(b), 12(b) respectively. Stretching models with higher values of $L(L=1.0$ and $L=1.5$ ) always predicted the anticyclonic ring to be larger than experimental observations and thus will not be presented here. For large values of the nondimensional time $t_{o b s}[R o]$ (see figure 11), good agreement is found between the diffusive model and the experimental measurements.

For moderate values of the non-dimensional observation time $t_{o b s}[R o]$ (see figure 12), the experimental radial profiles in the region above the rotating disk are underestimated by the inviscid model and overestimated by the diffusive model, as a consequence of the 'vortex compression' process mentioned above. The anticyclonic ring was formed after a transition through a baroclinically unstable stage, so that neither the stretching model nor the diffusive model are able to describe it. The stretching model, with $L=0.5$, does not fit the observations (the discrepancy is even larger with the other values of $L$ ), and the diffusive model predicts a very sharp vorticity profile close to the edge of the rotating disk, in contrast to the smooth experimental profile. Scrutinizing the shadowgraph images of the flow near the edge of the rotating disk indicates non-stationary flow and small-scale mixing for moderate values of $t_{o b s}[R o]$. 

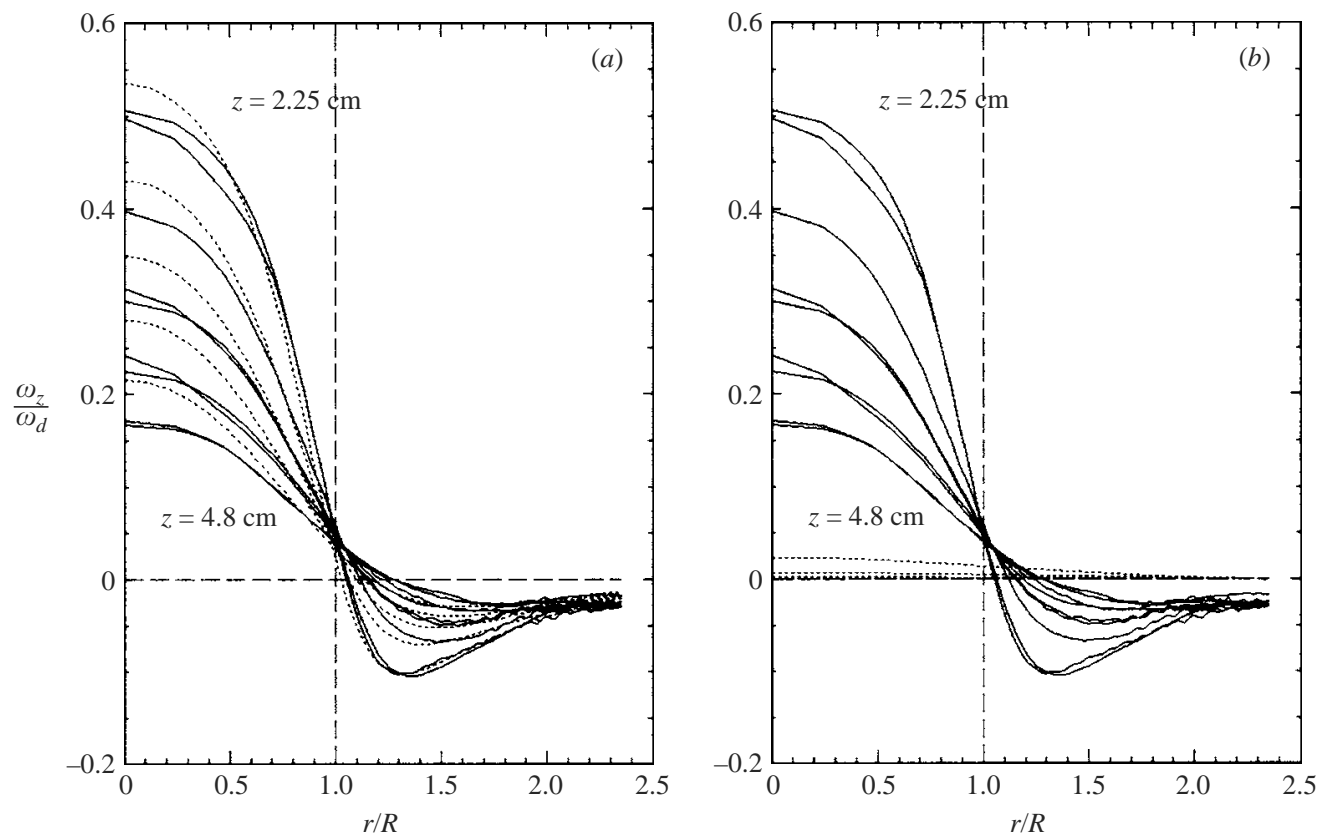

FIGURE 11. Radial profiles of non-dimensional vertical vorticity $\omega_{z} / \omega_{d}$ at different heights above the rotating disk. - The experimental curves based upon PIV measurements in horizontal planes $2.25,2.9,2.5,4.1$ and $4.8 \mathrm{~cm}$ above the rotating disk; $\cdots$, predictions by $(a)$ the diffusive model, and $(b)$ the stretching model for $L=0.5$. For experimental parameters, see table 4, experiment $c$.
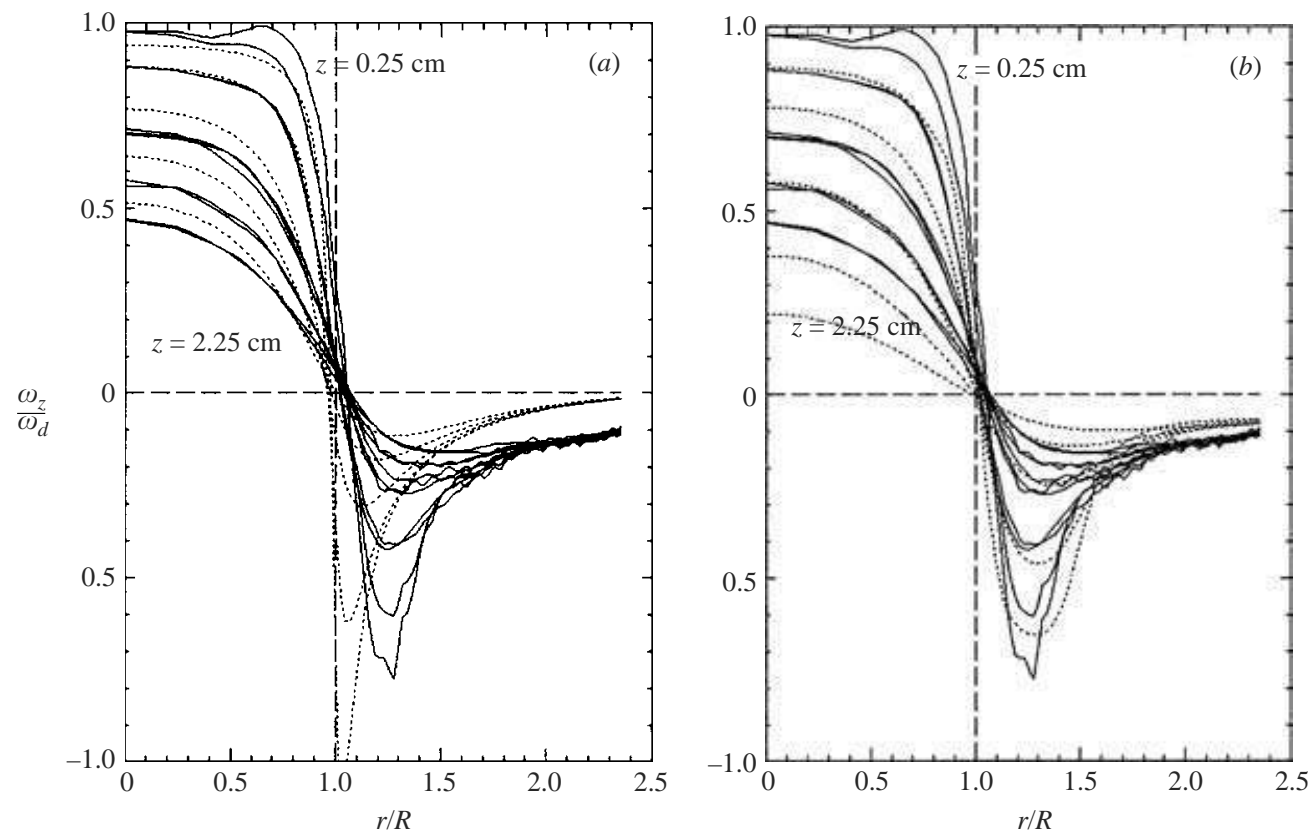

FIGURE 12. As figure 11 but for experiment $h$ and in horizontal planes 0.25, 0.5, 1.0, 1.6 and $2.25 \mathrm{~cm}$ above the rotating disk. 
Diffusive processes tend to sharpen the velocity profile near the edge of the disk and may lead to shear instabilities. The competition between the sharpening of the profile by diffusion and the smoothing associated with small-scale mixing would then explain the non-stationary flow observed in the experiments. Eventually, the fluid around the disk has spin-up so that the shear reduces and instabilities near the disk disappear.

\section{Concluding remarks}

We have investigated the spin-up of fluid by a cyclonically rotating disk in a rotating stratified fluid, for $N / f$-values larger than 1 and Rossby number around 1. We focused mainly on flows which remained stable during the front instability, and which returned eventually to an axisymmetric state.

We can summarize the spin-up process as follows: During an initial stage a meridional circulation is driven by the Ekman boundary layer, which draws fluid into the disk, and effluxes it into a circular density front at the disk edge, conservation of potential vorticity being responsible for the cyclonic and anticyclonic vorticity above the disk and density front, respectively. From the onset of the disk rotation, vertical vorticity diffuses vertically from the disk and eventually governs the subsequent flow stage. We found that vorticity diffusion dominates the flow evolution completely when the ratio, $\gamma$, between the diffusion timescale $\tau_{\text {diff }}$ and the spin up timescale $\tau_{s u}$, satisfies $\gamma<1$, i.e. when $E_{k}^{-1 / 2}(f / N)<3$, whereas the stretching process is recognized as a separate stage in the experiments only if $E_{k}^{-1 / 2}(f / N)>3$.

The spin-up time, found for the stretching model, $\tau_{s u}=1.2 /\left(E^{1 / 2} N\right)$, compares well with the spin-up time $\tau_{s u}=1.3 /\left(E^{1 / 2} N\right)$ found by Flór, Ungarish \& Bush (2002) for the spin-up from rest of a stratified fluid in a cylindrical container. Their flow differed from linear spin-up (see Walin 1969) in the formation of mixed corner regions and isodensity surfaces that are deflected downwards in the centre of the tank. In the present experiment, the front, resulting from the fluid ejected by the Ekman layer, is arrested around the deformation radius and may enhance a similar recirculation as in spin-up from rest. The model appears weakly affected by the radial extent of this front, $L$. For values of $L$ between $0.5 R$ and $1.5 R$, agreement with observations suggests a weak sensitivity of the spin-up of fluid above the disk to the growth of this density front. However, the development of the instability of the density front has an impact on the spin-up, especially beyond the disk edge, because of the non axisymmetric flow evolution.

\section{Appendix. Influence of the boundary conditions on solutions of the stretching model}

To solve (4.4) for the stretching model, boundary conditions are required, but the size and the shape of the negative vorticity ring observed in the experiments are a priori unknown. In $\S 4.1$, we assumed an anticyclonic ring with constant vorticity of radial extent $L$. For this particular shape, the non-dimensional form of the boundary conditions on the plane $z=0$ are

$$
\left.\begin{array}{l}
v_{\theta}(r, z=0)=r \quad \text { for } r \leqslant 1, \\
v_{\theta}(r, z=0)=\frac{1}{(1+L)^{2}-1}\left(-r+\frac{(L+1)^{2}}{r}\right) \quad \text { for } r \in[1, L], \\
v_{\theta}(r, z=0)=0 \quad \text { for } r \geqslant 1+L .
\end{array}\right\}
$$


To solve equation (4.4), we replaced it by a set of finite-difference equations on a regular grid of dimensions $[0,5] \times[0,5]$ with a resolution of $50 \times 50$, and solved it numerically by a relaxation method (see e.g. Press et al. 1992). To calculate the finitedifference derivative terms near the boundaries, we used equations (A 1) to prescribe values of $v_{\theta}$ on the lower boundary $(z=0)$, and we set $v_{\theta}$ equal to zero on the upper $(z=5)$ and vertical $(r=5$ and $r=0)$ boundaries. As an initial guess for $R o_{b}=0$, we used the solution of (4.5) obtained by Hankel transform of the boundary equations. We then increased $R o_{b}$ step by step, using the latter solution as an initial guess for the next value of $R o_{b}$. The Rossby number $R o_{b}$ was taken between 0 and 4.0, and the radial extent was $L=0.5,1.0$ and 1.5 .

The impact of $L$ on the flow pattern above the rotating disk was investigated, using vertical profiles of central vorticity like those plotted in figure 6 . The vertical size $H$ of the vortex was estimated, using the inverse value of the slope of these profiles, and its dependence on Rossby number, $R o_{b}$, for each value of $L$ leads to

$$
\left.\begin{array}{l}
H\left(R o_{b}\right)_{(L=0.5)}=\frac{f R}{N}\left(0.75+0.50 R o_{b}\right), \\
H\left(R o_{b}\right)_{(L=1.0)}=\frac{f R}{N}\left(0.8+0.58 R o_{b}\right), \\
H\left(R o_{b}\right)_{(L=1.5)}=\frac{f R}{N}\left(0.87+0.64 R o_{b}\right) .
\end{array}\right\}
$$

Equations (A 2) show a very weak dependence of the vertical extent $H$ on the radial extent $L$ in comparison with its dependence on Rossby number. Therefore, the equation for $L=1.0$ was used in the second part of $\S 4.1 .2$ to obtain an averaged definition of the inviscid spin-up time $\tau_{s u}$, infered from the vertical extent of the flow.

If we now consider radial profiles of vorticity in horizontal planes, such as those plotted in figures $11(b)$ and $12(b)$, the radial extent $L$ determines the size of the negative vorticity region in all the horizontal planes. Radial profiles of vertical vorticity vary from a step-like pattern on the disk surface, $z=0$, to a Gaussian profile, with smoother vorticity gradients at a distance above the disk. The position of the zero vorticity point remains close to the radius of disk for all $z$. As a consequence, variations of the flow characteristics in the vertical and radial directions are strongly dissociated, and only the radial direction is affected by the radial extent $L$ of the anticyclonic ring.

\section{REFERENCES}

BAKER, D. J. 1971 Density gradients in a rotating stratified fluid: experimental evidence for a new instability. Science. 172, 1029-1031.

BAKER, E. T. 1995 Characteristics of hydrothermal discharge following amagmatic intrusion. Hydrothermal Vents and Processes, Geol. Soc. Spec. Pub. 87, 65-76.

Bush, J. W. M. \& Woods, A. W. 1999 Vortex generation by line plumes in a rotating stratified fluid. J. Fluid Mech. 388, 289-313.

Calman, J. 1977 Experiments on high richardson number instability of a rotating stratified shear flow. Dyn. Atmos. Oceans 1, 277-297.

Clercx, H. J. H., Beckers, M., Verzicco, R. \& van Heisst, G. F. J. 2001 Dynamics of pancakelikevortices in a stratified fluid: experiments, model and numerical simulations. J. Fluid Mech. 433, $1-27$.

Davies, P. A., Guo, Y., Boyer, D. L. \& Folkard, A. M. 1995 The flow generated by the rotation of a horizontal disk in a stratified fluid. Fluid Dyn. Res. 17, $27-47$.

Drazin, P. G. \& ReID, W. H. 1981 Hydrodynamic Stability. Cambridge University Press. 
Duck, P. W. \& Foster, M. R. 2001 Spin-up of homogeneous and stratified fluids. Annu. Rev. Fluid Mech. 33, 231-263.

EADY, E. T. 1949 Long waves and cyclone waves. Tellus 1, 33-52.

Fincham, A. \& SpedDing, G. 1997 Low cost, high resolution DPIV for measurements of turbulent fluid flow. Exps. Fluids 23, 449-462.

Fincham, A. \& Delerce, G. 2000 Advanced optimization of correlation imaging velocimetry algorithms. Exps. Fluids 29, S013-S022.

FLIERL, G. R. \& Mied, R. P. 1985 Frictionally induced circulations and spin-down of a warm-core ring. J. Geophys. Res. 90, 8917-8927.

FLór, J.-B., Ungarish, M. \& Bush, J. W. M. 2002 Spin-up from rest in a stratified fluid: boundary flows. J. Fluid Mech. 472, 51-82.

Greenspan, H. P. 1968 The Theory of Rotating Fluids. Cambridge University Press.

Griffith, R. W. \& Linden, P. F. 1981 The stability of buoyancy-driven coastal currents. Dyn. Atmos. Oceans 5, 281-306.

Hide, R. \& Titman, C. W. 1967 Detached shear Layers in a rotating fluid. J. Fluid Mech. 29, 39-60.

Honji, H., Taneda, S. \& Tatsudo, M. 1980 Some practical details of the electrolytic precipitation method of flow visualisation. Res. Inst. Appl. Mech. Kyushu Univ. 28, 83-89.

Hopfinger, E. G., Flór, J.-B., Chomaz, J. M. \& Bonneton, P. 1991 Internal waves generated by a moving sphere and its wake in a stratified fluid. Exps. Fluids 11, 255-262.

Kloosterziel, R. 2000 Surface forced internal waves and vortices in uniformly stratified and rotating fluids. J. Fluid Mech. 421, 39-81.

Manley, T. O. \& Hunkins, H. 1985 Mesoscale eddies of the arctic ocean. J. Geophys. Res. 90 (C3), 4911-4930.

MCInTyre, M. E. 1970 Diffusive destabilization of the baroclinic circular vortex. Geophys. Fluid Dyn. 1, 19-57.

McWilliams, J. C. 1985 Submesoscale, coherent vortices in the ocean. Geophys. Rev. 23, 165-182.

Press, W. H., Teukolsky, S. A., Vetterling, W. T. \& Flannery, B. P. 1992 Numerical Recipes in Fortran, The Art of Scientific Computing, 2nd Edn. Cambridge University Press.

Spence, G. S. M., Foster, M. R. \& Davies, P. A. 1992 The transient response of a contained rotating stratified fluid to impulsively started surface forcing. J. Fluid Mech. 243, 33-50.

WaLIN, G. 1969 Some aspects of time-dependent motion of a stratified rotating fluid. J. Fluid Mech. 36, 289-307. 